\title{
Heavy Flavour Production and Decay with Prompt Leptons in the ALEPH Detector.
}

\section{The ALEPH Collaboration}

\begin{abstract}
In 431,000 hadronic $Z$ decays recorded with the ALEPH detector at LEP, the yields of electrons and muons in events with one or more prompt leptons have been analysed to give information on the production and decay of heavy quarks. The fractions of $b \bar{b}$ and $c \bar{c}$ events are measured to be $0.219 \pm 0.006 \pm 0.005$ and $0.165 \pm 0.005 \pm 0.020$, and the corresponding forward-backward asymmetries at $\mathrm{Z}$ mass are measured to be $0.090 \pm 0.013 \pm 0.003$ and $0.111 \pm 0.021 \pm 0.018$, after QED and QCD corrections. Measurements for the semileptonic branching ratios $\operatorname{Br}\left(b \rightarrow \ell^{-} \bar{\nu} \mathrm{X}\right)$ and $\operatorname{Br}\left(b \rightarrow c \rightarrow \ell^{+} \nu \mathrm{X}\right)$ yield $0.114 \pm 0.003 \pm 0.004$ and $0.082 \pm 0.003 \pm 0.012$, respectively. The dilepton events enable measurement of the $b$ mixing parameter, $\chi=0.114 \pm 0.014 \pm 0.008$. Results are also presented for the energy variation of the $b \bar{b}$ asymmetry and the parameters required to describe heavy quark fragmentation. From the asymmetry measurements, the effective electroweak mixing angle is $\sin ^{2} \theta_{W}^{e f f}=0.2333 \pm 0.0022$.
\end{abstract}




\section{Introduction}

Approximately $40 \%$ of the hadronic decays of the $Z$ boson are to pairs of $b$ and $c$ quarks. Isolation of clean samples of these final states allows one to probe the electroweak interaction in the quark sector. The basic electroweak parameters are the ratios of the decay widths for $b$ and $c$ decay to the total hadronic width and the forward-backward asymmetry for both species of heavy quark. The widths require an identification procedure (tag) which provides good separation of the $b, c$, and $u d s$ decays of the $\mathrm{Z}$ whilst the asymmetries additionally require a tag which discriminates the quark direction from the antiquark direction. Semileptonic decays of beauty and charm states produce prompt electrons and muons of high momentum due to the hard fragmentation of the heavy quark states and also, in the case of beauty, with high momentum perpendicular to the direction of the parent heavy flavour hadron. In this paper the $p$ and $p_{\perp}$ spectra of prompt electrons and muons are used to tag the $b$ and $c$ states; $p_{\perp}$ always refers to the momentum of the lepton perpendicular to the jet to which it belongs.

The number of prompt leptons in a sample of hadronic events is determined by the products

$$
\begin{gathered}
\operatorname{Br}\left(b \rightarrow \ell^{-} \bar{\nu} \mathrm{X}\right) \Gamma(b \bar{b}) / \Gamma(h a d), \\
\operatorname{Br}\left(b \rightarrow c \rightarrow \ell^{+} \nu \mathrm{X}\right) \Gamma(b \bar{b}) / \Gamma(h a d), \\
\text { and } \\
\operatorname{Br}\left(c \rightarrow \ell^{+} \nu \mathrm{X}\right) \Gamma(c \bar{c}) / \Gamma(h a d)
\end{gathered}
$$

The three processes $b \rightarrow \ell^{-}, b \rightarrow c \rightarrow \ell^{+}$, and $c \rightarrow \ell^{+}$are distinguished by their different spectra in the $\left(p, p_{\perp}\right)$ plane. The individual factors in the products can only be isolated by a simultaneous consideration of single and dilepton events, which in principle has the potential to extract all five quantities. However, the statistics of the dilepton sample are inadequate, particularly for both the cascade and charm decays, and so in this work the semileptonic charm decay rate is taken from previous measurements and the current data are used to measure the other four quantities.

The momentum spectrum of the leptons is strongly affected by the heavy quark fragmentation and so this allows a measurement of $\left\langle x_{b}\right\rangle$ and $\left\langle x_{c}\right\rangle$, where $x_{b / c}=E_{b / c}$ hadron $/ E_{b e a m}$, within the framework of a particular fragmentation model. A comparison of same and opposite charge dilepton events enables the integrated mixing parameter, $\chi$, to be determined.

The analyses require knowledge of the rest frame semileptonic decay spectra which is the main contributor to the transverse momentum lepton spectra. Where possible this is taken from published data with models used to extrapolate to regions for which no data exist. This is discussed in section 3. The momentum spectrum shape is largely determined by fragmentation; different models are considered and this is also discussed in section 3 . 
Clean identification of the prompt lepton signal is vital. Hadronic event selection, lepton identification in ALEPH and the optimisation of the definition of jets are described in detail in reference [1]. Lepton detection efficiencies and contamination rates are taken from the data, except for the contamination of the muon signal by $\pi$ and $\mathrm{K}$ decays in flight. The measured rates are then used to recalibrate the Monte Carlo; this will be referred to as the corrected Monte Carlo in the following.

The results are presented in section 4 . This commences with a number of analyses purely in the $b$ sector where a cut at high $p_{\perp}$ is used to give a relatively pure $b$ sample. Using this cut, values are obtained for the $b \bar{b}$ fraction of hadronic events, $R_{b}$, the forward-backward asymmetry of $b \bar{b}$ production, $A_{F B}^{b}$, the $b$ mixing parameter, $\chi$, and the $\operatorname{Br}\left(b \rightarrow \ell^{-} \bar{\nu} \mathrm{X}\right)$. The energy dependence of the forwardbackward asymmetry is also presented with the high $p_{\perp}$ sample. Extension to lower $p_{\perp}$ where the charm component becomes significant requires a simultaneous fit over the lepton and dilepton spectra. This gives the $c \bar{c}$ fraction of hadronic events, $R_{c}$, the cascade branching ratio $\operatorname{Br}\left(b \rightarrow c \rightarrow \ell^{+} \nu \mathrm{X}\right)$, and $A_{F B}^{c}$ as well as the previous quantities and also allows measurement of the mean fragmentation parameters for beauty and charm. A detailed discussion of the systematic uncertainties is given in section 4.1.

Throughout this paper, unless specified otherwise, charge conjugate reactions are implied, and $b \rightarrow \ell^{-}, c \rightarrow \ell^{+}$, etc. will refer to the decays $b \rightarrow \ell^{-} \bar{\nu} \mathrm{X}, c \rightarrow \ell^{+} \bar{\nu}$ $\mathrm{X}$, etc. . The symbol $\ell$ indicates either electrons or muons, but not the sum of the two.

\section{The Aleph detector}

The ALEPH detector has been described in detail elsewhere [2]. For the data used in these analyses, taken in 1990 and 1991, charged tracks are measured over the range $|\cos (\theta)|<0.95$, where $\theta$ is the polar angle, by an inner cylindrical drift chamber (ITC) and a large cylindrical time projection chamber (TPC). These chambers are immersed in a magnetic field of 1.5 Tesla and together measure the momentum of charged particles with a resolution [3] of

$$
\delta P / P=0.0008 P \quad(P \text { in } G e V / c)
$$

The TPC provides up to 330 measurements of the specific ionization, $\mathrm{d} E / \mathrm{d} x$, of each charged track. Outside the TPC is the electromagnetic calorimeter (ECAL), which is constructed of 45 layers of lead interleaved with proportional wire chambers. The ECAL has an energy resolution of

$$
\delta E / E=0.19 / \sqrt{E}+0.01 \quad(E \text { in } G e V)
$$

and is used together with the $\mathrm{d} E / \mathrm{d} x$ measurements of the TPC to identify electrons. The hadron calorimeter ( $\mathrm{HCAL}$ ) is the iron of the magnet return yoke interleaved 
with 23 layers of streamer tubes which provide a two dimensional view of the development of hadronic showers. The HCAL is used in conjunction with the muon chambers and the tracking detectors to identify muons. The calorimeters and muon chambers cover nearly the entire $4 \pi$ solid angle.

\section{The simulation of heavy flavour processes in $\mathrm{Z}$ decays at LEP}

\subsection{The HVFL program}

For heavy flavour studies ALEPH has developed a program, HVFL, based on JETSET 7.3 [4]. JETSET procedures are used for the parton shower and string fragmentation with parameters tuned to fit event shape variables [5] and to take account of final state radiation. In addition several modifications have been made to increase flexibility and further improve agreement with known results. These are:

- The process $e^{+} e^{-} \rightarrow q \bar{q}$ is generated with DYMU2 [6] to give the best possible calculation of the initial state photon radiation.

- The decay channels of charm hadrons take into account the latest experimental results for both exclusive and inclusive modes [7].

- Two body branching ratios of the $b$ mesons measured by ARGUS and CLEO [8] are used. Unmeasured two body decays are computed from the measured ones using the Stech-Bauer approach [9].

- B meson decays to baryons are added so as to reproduce measurements of inclusive production [10].

- The decay chain for $\mathrm{B} \rightarrow \mathrm{J} / \psi+\mathrm{X}$ has been modified to give multibody decays and a $\mathrm{J} / \psi$ spectrum which agrees with data. $\mathrm{B}$ meson decays to $\psi^{\prime}$ have been added with a correct simulation of the $\psi^{\prime} \rightarrow J / \psi \pi \pi$ decay.

- The basic dynamics for $\mathrm{B} \rightarrow l \nu \mathrm{X}$ has been modified. The decays $\mathrm{B} \rightarrow l \nu \mathrm{D}$ and $\mathrm{B} \rightarrow l \nu \mathrm{D}^{*}$ in the ratio $1: 3$ are implemented according to the Korner-Schuler model [11]. Sufficient higher mass contributions, $\mathrm{B} \rightarrow l \nu \mathrm{D}^{* *}$ and $\mathrm{B} \rightarrow l \nu \mathrm{D}^{*} \pi$, are included so that HVFL approximately reproduces the lepton energy spectrum fitted by ARGUS and CLEO [12] using the model of Altarelli et al. (ACCMM) [13]. In practice the final states $\mathrm{D}: \mathrm{D}^{*}: \mathrm{D}^{* *}: \mathrm{D}^{*} \pi$ are generated in the ratios $0.211: 0.639: 0.075: 0.075$.

- Final states resulting from $b \rightarrow u$ transitions are introduced. They are computed in the free quark model with a rate proportional to phase space. 


\subsection{Corrections for decay and fragmentation models}

To further improve the description of the lepton spectra, the simulated events are given a weight based on the lepton energy in the $b$ hadron centre of mass to account for the following:

- The $b \rightarrow \ell^{-}$spectrum. Models of the $b$ semileptonic decay differ in their treatment of the higher mass $\mathrm{D}^{* *}$ and $\mathrm{D}^{*} \pi$ components. Fitting the available data $[12,14]$, to models with large explicit $\mathrm{D}^{* *}$ contributions such as that of Isgur et al. [15] (ISGW ${ }^{* *}$ ) yields softer lepton spectra than with the inclusive (ACCMM) model. For the analyses the two approaches are taken as extremes and weights used so that both models reproduce the CLEO fits. The quoted results are the average of these two with an assigned modelling uncertainty of half the difference.

- The $c \rightarrow \ell^{+}$spectrum. The lepton energy spectrum in the $c$ hadron rest frame from charm decays contains large uncertainties. The main source of experimental information is from DELCO [16]. In that experiment $\psi^{\prime \prime}$ decays are the source of $D^{0}$ and $D^{+}$with approximately the same production rate, except for a small phase space effect. The shape of the energy spectrum generated in JETSET is softer than the DELCO results and is weighted to reproduce it. Half of the difference between the weighted and unweighted results is taken as the modelling uncertainty.

- The $b \rightarrow c \rightarrow \ell^{+}$spectrum. This is a two step process and the experimental situation is less clear. For the analyses the energy spectrum is taken directly from JETSET but the full difference between these results and those using the weights for the $c \rightarrow l \nu \mathrm{X}$ are taken as the modelling uncertainty.

- Internal bremsstrahlung in $b$ and $c$ semileptonic decays. The PHOTOS Monte Carlo [17] is used to give the ratio of the lepton spectra with and without internal bremsstrahlung and this is then parametrised as a function of the lepton rest frame energy to give the weight. The procedure is approximate as it only corrects the lepton energy and not the direction but this has a negligible effect on the results. The main effect is a $4 \%$ correction to the $b \rightarrow e^{-}$branching ratio.

- Heavy Quark fragmentation. The events are generated with both $b$ and $c$ fragmentation described by the Peterson et al. form [18] (PSSZ) which is defined in terms of the variable $z$, denoting the fraction of $\left(E+P_{\|}\right)$taken by the heavy flavour hadron. It depends upon one parameter, $\varepsilon_{Q}$, for each quark. The effects of the alternative fragmentation scheme of Kartvelishvili et al. [19] (KLP) are investigated by weighting the generated events in terms of their $z$ value. 


\section{Data analysis}

Prompt electron and muon candidates result from the following physical processes:

- Primary semileptonic decays of $b$ hadrons, denoted $b \rightarrow \ell^{-}$.

- Decays of a $\tau$ from a $b$ decay, denoted $b \rightarrow \tau \rightarrow \ell^{-}$.

- Cascade decays from the charm daughter of a $b$ parent, denoted $b \rightarrow c \rightarrow \ell^{+}$.

- Cascade decays of a charm state from the $\mathrm{W}^{-}$in the $b$ decay, denoted $b \rightarrow(\bar{c} s) \rightarrow \ell^{-}$.

- Semileptonic decays of charm states produced in $\mathrm{Z} \rightarrow c \bar{c}$, denoted $c \rightarrow \ell^{+}$.

- Leptons from non-prompt sources or hadrons misidentified as leptons, denoted fake.

The degree to which the origin of the observed leptons must be classified depends on the physical quantity to be measured. For mixing and asymmetry the sign is crucial and therefore the $b \rightarrow c \rightarrow \ell^{+}$cascade decay is an important contributor to the background together with the charm component. They are approximately of the same size in the lepton sample while in the dilepton sample the charm component is suppressed. On the other hand for the $\mathrm{Z}$ width to $b \bar{b}$ no information on the quark sign is needed so the primary charm component is the most significant background. Separation is achieved on a statistical basis by the use of the $\left(p, p_{\perp}\right)$ spectrum; leptons from primary $b$ decay have relatively high values for both $p$ and $p_{\perp}$ as a result of the hard heavy quark fragmentation and the high $b$ hadron mass respectively. Below $3 \mathrm{GeV} / \mathrm{c}$ some muons do not reach the muon chambers and so the identification efficiency falls off. Consequently, a minimum momentum cut of $3 \mathrm{GeV} / \mathrm{c}$ is applied for all lepton candidates. Electron identification is very good down to much lower momenta but there is then a large background of non-prompt electrons from conversions.

The choice of axis for the determination of the transverse momentum is important. In reference [1] it is shown that, with the data available from the ALEPH detector, the best discrimination is achieved when both neutrals and charged particles are used for the jet analysis and the jet axis is redefined after the lepton has been excluded from the jet.

Results are obtained with two different techniques which are compared for consistency. In the first, the high $p_{\perp}$ analyses, a lower cut is made on the lepton transverse momentum at $1.25 \mathrm{GeV} / \mathrm{c}$ to produce a relatively pure sample of primary $b$ decays. The predicted sample compositions for both electrons and muons are given in table 1 . The choice of $1.25 \mathrm{GeV} / \mathrm{c}$ is a compromise between sample purity and adequate statistics. Measurements made on this sample of events 
are essentially counting experiments and are purely in the $b$ sector. Events are categorized depending on whether they are single or dilepton, and in the latter case, with regard to the relative charges and directions of the two leptons. Estimated corrections for contaminants from background, cascade and charm decays are then subtracted. The high $p_{\perp}$ analyses determine $R_{b}, \operatorname{Br}\left(b \rightarrow \ell^{-} \bar{\nu} \mathrm{X}\right), A_{F B}^{b}$ and $\chi$. Choosing this restricted region leads to relatively simple equations in which the effects of the backgrounds from lepton misidentification and leptons from other sources are small. However, the magnitude of the contamination from other sources does depend on external measurements of the branching ratios and theoretical predictions for the spectra.

\begin{tabular}{|l||c|c|c|}
\hline \multicolumn{1}{|c||}{ Event type } & \multicolumn{3}{c|}{ Sample fraction (\%) } \\
\cline { 2 - 4 } & $e$ & $\mu$ & Total \\
\hline$b \rightarrow \ell^{-}$ & 83.2 & 73.6 & 77.2 \\
$b \rightarrow \tau \rightarrow \ell^{-}$ & 1.1 & 1.0 & 1.0 \\
$b \rightarrow c \rightarrow \ell^{+}$ & 4.8 & 5.1 & 5.0 \\
$b \rightarrow(\bar{c} s) \rightarrow \ell^{-}$ & 0.3 & 0.3 & 0.3 \\
$c \rightarrow \ell^{+}$ & 6.0 & 6.5 & 6.3 \\
$K, \pi \rightarrow \mu$ & - & 4.6 & 2.9 \\
photon conversions & 1.5 & - & 0.6 \\
misid. hadron & 1.0 & 7.2 & 4.8 \\
other sources & 2.1 & 1.7 & 1.9 \\
\hline
\end{tabular}

Table 1: Sample composition for the two lepton species and for the total sample, with $p>3 \mathrm{GeV} / \mathrm{c}$ and $p_{\perp}>1.25 \mathrm{GeV} / \mathrm{c}$. "Other sources" include $\mathrm{J} / \psi$ and are predominantly from $b \bar{b}$ events. When there is more than one lepton in the same event, the one with the highest $p_{\perp}$ is used for this table.

Further information may be obtained by means of a detailed fit to the $\left(p, p_{\perp}\right)$ lepton spectra over the full $p_{\perp}$ range. This is performed in the second analysis, referred to as the global analysis. In the low $p_{\perp}$ range, there are many leptons from direct charm and cascade $b$ decays which enable quantities in both the $b$ and $c$ sectors to be measured. Present statistics do not merit simultaneously fitting the $b \rightarrow \ell^{-}, b \rightarrow c \rightarrow \ell^{+}$or $c \rightarrow \ell^{+}$branching ratios as discrimination between $b \rightarrow c \rightarrow \ell^{+}$decays, $c \rightarrow \ell^{+}$decays and the background in the low $p_{\perp}$ region is not great. As the $c \rightarrow \ell^{+}$branching ratio is better known than the $b \rightarrow c \rightarrow \ell^{+}$ one, the latter is chosen to be measured and the former is taken from low energy measurements.

All the analyses suffer from imperfect knowledge of the total rates and shape of the lepton spectra from $b \rightarrow \ell^{-}, b \rightarrow c \rightarrow \ell^{+}$, and $c \rightarrow \ell^{+}$decays. In the global analysis, the rates, except for $\operatorname{Br}\left(c \rightarrow \ell^{+} \nu \mathrm{X}\right)$, are fitted; in the high $p_{\perp}$ 
analyses the $b \rightarrow \ell^{-}$and $b \rightarrow c \rightarrow \ell^{+}$rates are input where appropriate from the global fit. The procedure adopted to assess the uncertainties in the results arising from the sensitivity to the shape of the spectra is described in section 3.2. The effects are different for the two forms of analysis. For the high $p_{\perp}$ analyses, the measurements must be extrapolated using the model into the low $p_{\perp}$ region. For the global analysis, the functions which are used in the fit are changed according to the model.

\subsection{Sources of systematic uncertainties}

All of the analyses are subject to uncertainties inherent in lepton identification and the modelling of lepton production. Those uncertainties specific to an individual analysis are discussed with the appropriate results.

Identification uncertainties for electrons. For electrons the efficiencies of both the ECAL and $\mathrm{d} E / \mathrm{d} x$ identification are directly measured from the data with good statistics [1]. A total uncertainty of $3 \%$ is set for the electron identification efficiency. The probability of hadron misidentification is directly measured on data; an uncertainty of $10 \%$ is assumed.

The rate of electrons from photon materialization is measured on data by the number of pairs observed with at least one track consistent with the electron identification criteria. The efficiency of the pair finder is known to $10 \%$.

Identification uncertainties for muons. From the studies of the processes $\mathrm{Z} \rightarrow \mu^{+} \mu^{-}$and $\tau$ decays, as described in [1], a global uncertainty on the muon identification efficiency has been set at $3 \%$. The contamination from hadron punch-through is determined from pure samples of hadrons selected from $\tau$ decays (using the channels $\left.\tau \rightarrow \rho \nu, \tau \rightarrow K^{*} \nu, \tau \rightarrow \pi \pi \pi \nu\right)$ and $K^{0}$ decays $\left(K^{0} \rightarrow \pi \pi\right)[1]$; this also allows checks for the hadron decays. From this analysis, uncertainties on the punch-through and decay rate of $20 \%$ and $10 \%$, respectively have been assigned.

The rate of $b \rightarrow(\bar{c} s) \rightarrow \ell^{-}$. The rate of lepton production from $b \rightarrow(\bar{c} s) \rightarrow \ell^{-}$ is taken from phase space calculations [20] and is equal to $14 \%$ of the rate of $b \rightarrow c \rightarrow \ell^{+}$. A $50 \%$ uncertainty is set on this number to compute systematic uncertainties.

$b \rightarrow \tau$ branching ratio. For this branching ratio the measured value [21] of $(4.08 \pm 0.76) \%$ has been used. The systematic uncertainties of this measurement were not used in estimating the error due to $b \rightarrow \tau$ because they are explicitly calculated in this paper. 
$\mathbf{J} / \psi$ production from $\mathbf{B}$ decays. The $\mathrm{B} \rightarrow \mathrm{J} / \psi+\mathrm{X}$ branching ratio is assumed to be $1.12 \%$, with a $15 \%$ variation.

$b \rightarrow u$ transition. It is assumed that $3 \%$ of the $b$ quarks decay through $b \rightarrow u$. A $50 \%$ uncertainty is taken on this number.

The product of $R_{c} \cdot \operatorname{Br}\left(c \rightarrow \ell^{+}\right)$. The semileptonic branching ratio of charm decays is taken to be $(9.8 \pm 0.5) \%$ [22]. An uncertainty of $10 \%$ is assumed on $R_{c} \cdot \operatorname{Br}\left(c \rightarrow \ell^{+}\right)$for the high $p_{\perp}$ analyses, with $R_{c}=0.174$. For the global analysis, $R_{c}$ is a fitted variable, and $\operatorname{Br}\left(c \rightarrow \ell^{+}\right)$is varied by one $\sigma$.

Gluon splitting to heavy quarks. Charm and beauty quark pairs may be produced out of the vacuum. There is no experimental data on the rate at which this process occurs. An uncertainty of $100 \%$ on the JETSET predictions has been used.

Effect of heavy quark fragmentation modelling. In the overall fit a single parameter is used for each heavy quark species to describe the data with a fragmentation function of the PSSZ type. These parameters are free in the fit. To examine the sensitivity to an alternative fragmentation model, the fit has been repeated, weighting the events as a function of $z$ to give the KLP parametrisation. This also has one free parameter per species. The effect is negligible for all results except $R_{c}$, which changes by $3 \%$.

The estimate of uncertainties due to the modelling of $b \rightarrow \ell^{-}, b \rightarrow c \rightarrow \ell^{+}$, and $c \rightarrow \ell^{+}$decays have been discussed in section 3.2. For the high $p_{\perp}$ analyses, the value of $\operatorname{Br}\left(b \rightarrow c \rightarrow \ell^{+}\right)$is taken from the global fit.

\section{$5 \quad$ High $p_{\perp}$ analyses}

Using leptons with $p_{\perp}$ over $1.25 \mathrm{GeV} / \mathrm{c}$, the fraction of $\mathrm{Z} \rightarrow b \bar{b}$ in $\mathrm{Z}$ hadronic decays, $R_{b}$, the semileptonic branching ratio of the $b$, the $\mathrm{B}-\overline{\mathrm{B}}$ mixing and the forward-backward asymmetry of $b \bar{b}$ production from $Z$ decay have been measured.

\subsection{Measurement of $R_{b}$}

This method uses single and double tagged events to eliminate the uncertainties on the details of $b$ decays and fragmentation. Events with high $p_{\perp}$ leptons are split into two hemispheres with respect to the thrust axis, which is required to be within $|\cos \theta|<0.9$. They are then divided into two categories: a double tagged sample in which both hemispheres contain at least one lepton, and a single tagged 
sample in which one of the hemispheres does not contain a lepton. The value of $R_{b}$ is then derived from counting the numbers $N_{s t}$ and $N_{d t}$ of single tagged and double tagged events. These two numbers are related by:

$$
\begin{array}{ccc}
N_{s t}= & 2 P_{b}\left(1-C P_{b}\right) N_{b \bar{b}} & +N_{s t}^{\text {light }} \\
N_{d t}= & C P_{b}^{2} N_{b \bar{b}} & +N_{d t}^{\text {light }}
\end{array}
$$

Where:

- $N_{b \bar{b}}$ and $P_{b}$ are the two unknowns. $N_{b \bar{b}}$ is the number of $\mathrm{Z} \rightarrow b \bar{b}$ events in the hadronic sample. $P_{b}$ is the probability to tag one hemisphere of a $b \bar{b}$ event. This quantity contains all the uncertainties related to decay modelling, branching ratios, and fragmentation in the $b$ sector.

- $C=P_{b \bar{b}} / P_{b}^{2}$ where $P_{b \bar{b}}$ is the probability to tag the two hemispheres in a $b \bar{b}$ event. This factor accounts for possible correlations between the tagging efficiencies of the two hemispheres.

- $N_{s t}^{\text {light }}$ and $N_{d t}^{\text {light }}$ are the number of single and double tagged $u d s c$ events respectively.

The values of $C, N_{s t}^{\text {light }}$ and $N_{d t}^{\text {light }}$ are estimated from the corrected Monte Carlo (i.e. after the misidentification rates have been recalibrated from data) [1].

Computation of $C$. The $C$ factor has been been estimated using 264,739 fully simulated $b \bar{b}$ events. The value $C=1.002 \pm 0.012$ is consistent with one. There is no evidence that $C$ depends on the $p_{\perp}$ cut and hence it is independent of the physical origin of the leptons.

Results. Following the $\left|\cos \theta_{\text {thrust }}\right|<0.9$ cut, there are 380,604 hadronic $Z$ decays and 76,651 of these have identified leptons with $p>3 \mathrm{GeV} / \mathrm{c}$. For the $p_{\perp}$ cut at $1.25 \mathrm{GeV} / \mathrm{c}$, there are 16,241 single tag and 710 double tag events, as shown in table 2. The corrected Monte Carlo predicts that 2,158 single and 2 double tag events are expected to come from light quarks. Solving equations 1 and 2 with these light quark contributions subtracted yields $R_{b}=0.2215 \pm 0.0078$, where the uncertainty is statistical. The corresponding value of $P_{b}$ is $0.091 \pm 0.003$. A correction was made for the efficiency difference in the hadronic event selection between $b \bar{b}$ events and events of other flavours. The values of $R_{b}$ as a function of the $p_{\perp}$ cut are given in figure 1. There is a shift downward at lower values of $p_{\perp}$, but smaller than the systematic uncertainty, which dominates in this region.

As this method is independent of all aspects of the $b$ fragmentation model, $b$ decay models and experimental tagging, there are few sources of systematic uncertainties. These are given in table 3 . 

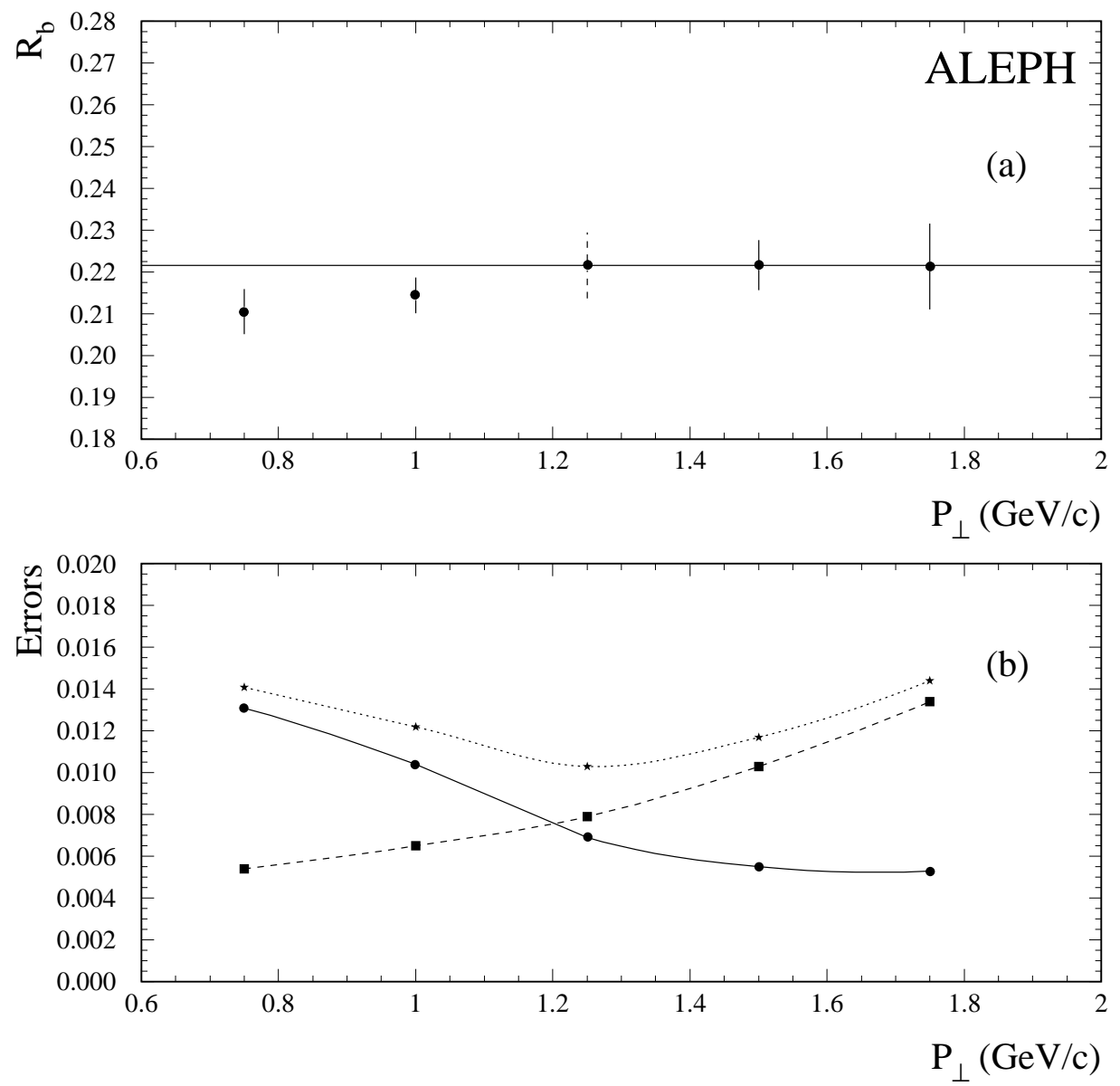

Figure 1: (a) $R_{b}$ variation with $p_{\perp}$ cut; Uncorrelated statistical errors are shown except for $1.25 \mathrm{GeV} / \mathrm{c}$ where the full statistical error is plotted. (b) The full line gives the systematic uncertainty variation with $p_{\perp}$, the dashed line is the statistical error and the dotted line is the total uncertainty. 


\begin{tabular}{|c|r|r|r|}
\hline$N_{\text {lep }}$ & 0 tag & 1 tag & 2 tags \\
\hline \hline 1 & 54087 & 12668 & \\
2 & 5258 & 3211 & 606 \\
3 & 343 & 329 & 95 \\
4 & 12 & 33 & 9 \\
\hline total & 59700 & 16241 & 710 \\
\hline
\end{tabular}

Table 2: Number of single and double tagged events with $p_{\perp} \geq 1.25 \mathrm{GeV} / \mathrm{c}$, as a function of the number of identified leptons in an event, $N_{l e p}$. The events are classified in three categories: the ' 0 tag' are events where all leptons fail the cuts, the ' 1 tag' are event where only one hemisphere is tagged and ' 2 tags' are events where the two hemispheres are tagged.

\begin{tabular}{|c|c|c|}
\hline Source & Variation & $\Delta R_{b}$ \\
\hline Monte Carlo statistics & $1 \sigma$ & 0.0016 \\
\hline$R_{c} \cdot \operatorname{Br}\left(c \rightarrow \ell^{+}\right)$ & $10 \%$ & 0.0036 \\
\hline$\varepsilon_{c}$ & $1 \sigma$ & 0.0013 \\
\hline Lepton ID efficiency & $3 \%$ & 0.0012 \\
\hline photon conversions & $10 \%$ & 0.0001 \\
\hline electron background & $10 \%$ & 0.0002 \\
\hline punch-through & $20 \%$ & 0.0024 \\
\hline decaying hadrons & $10 \%$ & 0.0012 \\
\hline$C=\frac{P_{b \bar{b}}}{P_{h}^{2}}$ & $1 \sigma$ & 0.0028 \\
\hline Selection correction & $1 \sigma$ & 0.0009 \\
\hline \multicolumn{2}{|l|}{$\begin{array}{l}\text { TOTAL } \\
\end{array}$} & 0.0059 \\
\hline
\end{tabular}

\begin{tabular}{|l|c|}
\hline Modelling uncertainty & $\Delta R_{b}$ \\
\hline$c \rightarrow \ell^{+}$model & 0.0030 \\
\hline
\end{tabular}

Table 3: Systematic uncertainties on $R_{b}$. 
At the present level of statistics, the $p_{\perp}$ cut at $1.25 \mathrm{GeV} / \mathrm{c}$ yields the smallest overall uncertainty. As statistics increase, the $p_{\perp}$ cut may be increased to reduce the systematic error. Taking into account all uncertainties and corrections for charm and lighter quarks, this method yields

$$
R_{b}=0.2215 \pm 0.0078(\text { stat }) \pm 0.0059(\text { syst }) \pm 0.0030 \text { (models) }
$$

\subsection{Measurement of $\operatorname{Br}\left(b \rightarrow \ell^{-} \bar{\nu} \mathrm{X}\right)$}

The semileptonic branching ratio, $\operatorname{Br}\left(b \rightarrow \ell^{-}\right)$, of $b$ hadrons is easily measured in the high $p_{\perp}$ region where contamination from other sources is low. The branching ratio is essentially the ratio of dilepton to single lepton events, after contamination from non-semileptonic $b$ decays has been removed and detection efficiencies have been accounted for.

Events where two leptons are detected in opposite hemispheres are mostly composed of events where both $b$ hadrons decayed semileptonically. The second largest component to this sample are events where one $b$ hadron decayed semileptonically and the other decayed through the cascade process $b \rightarrow c \rightarrow \ell^{+}$. Events where two leptons are detected in the same hemisphere are mostly composed of events where a $b$ hadron decayed semileptonically and the resulting $c$ hadron also decayed semileptonically. Thus, the same side dilepton sample depends on the product $\operatorname{Br}(b \rightarrow \ell) \operatorname{Br}(b \rightarrow c \rightarrow \ell)$ and is used to subtract the largest background to the opposite side dilepton sample.

The number of semileptonic $b$ decays is twice the number of $b \bar{b}$ events times the semileptonic branching ratio; the number of events in which there are two semileptonic $b$ decays is just the number of $b \bar{b}$ events times square of the branching ratio. From this, allowing for backgrounds and efficiencies, it follows that the branching ratio $\operatorname{Br}\left(b \rightarrow \ell^{-}\right)$is given by

$$
\operatorname{Br}\left(b \rightarrow \ell^{-}\right)=\frac{\left(\mathcal{D}^{\longleftrightarrow}-\mathcal{D}^{\Longrightarrow} \mathcal{F}^{\Longrightarrow}\right) \mathcal{F}^{\longleftrightarrow} / \epsilon_{\ell \ell}}{\mathcal{N} \mathcal{F} / \epsilon_{\ell}}
$$

where:

- $\mathcal{N}$ is the number of high $p_{\perp}$ leptons,

- $\mathcal{D} \Longrightarrow$ is the number of oppositely charged pairs of leptons less than $90^{\circ}$ apart. Same direction, same charge pairs are not considered because they provide little information about the background.

- $\mathcal{D} \longleftrightarrow$ is the number of pairs of leptons more than $90^{\circ}$ apart. There is no requirement on the charges of this sample in order to be independent of mixing effects. 
and the purity and efficiency factors are

- $\mathcal{F}$, the fraction of $\mathcal{N}$ which is due to semileptonic $b$ decays.

- $\mathcal{F} \Longrightarrow$, the fraction of $\mathcal{D} \Longrightarrow$ which have a $b$ that decays semileptonically and produce a $c$ hadron that also decays semileptonically.

- $\mathcal{F} \longleftrightarrow$, a correction factor for backgrounds to the opposite side dilepton sample other than $(b \rightarrow \ell)(b \rightarrow c \rightarrow \ell)$, i.e. a fake, converted pair, $\mathrm{J} / \psi$, etc. on one side of the event, with a semileptonic $b$ decay on the other side of the event.

- $\epsilon_{\ell}$ and $\epsilon_{\ell \ell}$ are the efficiencies to detect leptons from semileptonic decays in the $\mathcal{N}$ and $\mathcal{D} \longleftrightarrow$ cases.

Events with more than two leptons are used to create all the single lepton and dilepton combinations possible. So for example, a three lepton event will contribute three leptons to the lepton sample and three pairs to the dilepton samples.

The backgrounds in $\mathcal{N}$ are predominantly leptons from $b \rightarrow c \rightarrow \ell^{+}$and $c \rightarrow \ell^{+}$. The calculation of the purity factors $(\mathcal{F}, \mathcal{F} \Longrightarrow$, and $\mathcal{F} \longleftrightarrow)$ allows for the model dependencies described above; $\mathcal{F}$ ranges from 0.748 to $0.763, \mathcal{F} \Longrightarrow$ ranges from 0.508 to 0.516 , and $\mathcal{F} \longleftrightarrow$ is typically between 0.868 and 0.872 . There are also contaminants from lepton misidentification, and the rates of these processes are taken from the data, using the methods described in reference [1].

The backgrounds in $\mathcal{D} \Longrightarrow$ are predominantly from $\mathrm{J} / \psi$, which at LEP is produced from $b$ decay. The contribution to the total uncertainty from the $\mathrm{B} \rightarrow \mathrm{J} / \psi+\mathrm{X}$ branching ratio is however small.

Cascade decays $b \rightarrow(\bar{c} s) \rightarrow \ell^{-}$occur at a lower rate than $b \rightarrow c \rightarrow \ell^{+}$, and they have a softer $p_{\perp}$ spectrum. Such decays contribute to $\mathcal{D} \Longrightarrow$ only when both the $c$ and $(\bar{c} s)$ decay semileptonically, but can contribute to $\mathcal{D}^{\longleftrightarrow}$ when either decays semileptonically. At $p_{\perp}$ of $1.25 \mathrm{GeV} / \mathrm{c}$ or more however, $b \rightarrow(\bar{c} s) \rightarrow \ell^{-}$ makes a very small contribution to the dilepton samples (see table 7 ) and this correction is unimportant.

The ratio $\epsilon_{\ell \ell} / \epsilon_{\ell}^{2}$ is $1.073 \pm 0.019$, from a Monte Carlo study using about 13000 events with semileptonic decays on both sides. ${ }^{1}$ With this substitution, the measured branching ratio is seen to be inversely proportional to the efficiency. The ISGW ${ }^{* *}$ model predicts a softer $p_{\perp}$ spectrum than the ACCMM model, and consequently, the efficiency to detect semileptonic $b$ decays after a $p_{\perp}$ cut is lower in the ISGW ${ }^{* *}$ model by $9 \%$, and this is reflected in the second part of table 5 .

\footnotetext{
${ }^{1}$ The thrust axis cut at 0.9 is not used in this analysis.
} 
Results. The data sample and the Monte Carlo estimates of its composition are

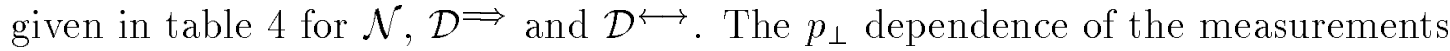
of $\operatorname{Br}\left(b \rightarrow \ell^{-}\right)$is shown in figure 2 ; little dependence on the $p_{\perp}$ cut exists.

\begin{tabular}{|c|c|r|}
\hline Leptons & Total & 19419 \\
$\mathcal{N}$ & $b \rightarrow \ell^{-}$ & $74.8 \%$ \\
& Other & $25.2 \%$ \\
\hline Same Side & Total & 211 \\
Lepton Pairs & $b \rightarrow\left(c \ell^{-}\right) ; c \rightarrow \ell^{+} \mathrm{X}$ & $51.2 \%$ \\
$\mathcal{D}^{\Longrightarrow}$ & $\mathrm{J} / \psi \rightarrow \ell^{+} \ell^{-}$ & $34.1 \%$ \\
& Other & $24.2 \%$ \\
\hline Opposite Side & Total & 768 \\
Lepton Pairs & {$[b \rightarrow \ell][b \rightarrow \ell]$} & $76.0 \%$ \\
$\mathcal{D}^{\longleftrightarrow}$ & {$[b \rightarrow \ell][b \rightarrow c \rightarrow \ell]$} & $12.8 \%$ \\
\multicolumn{2}{|c|}{ Other } & $11.2 \%$ \\
\hline
\end{tabular}

Table 4: Data sample and Monte Carlo composition for $p_{\perp}>1.25 \mathrm{GeV} / \mathrm{c}$. When there is more than one lepton in a hemisphere, all are used for this table. In the opposite side sample, $b \rightarrow c \rightarrow \ell$ includes $b \rightarrow \bar{c} s \rightarrow \ell$.

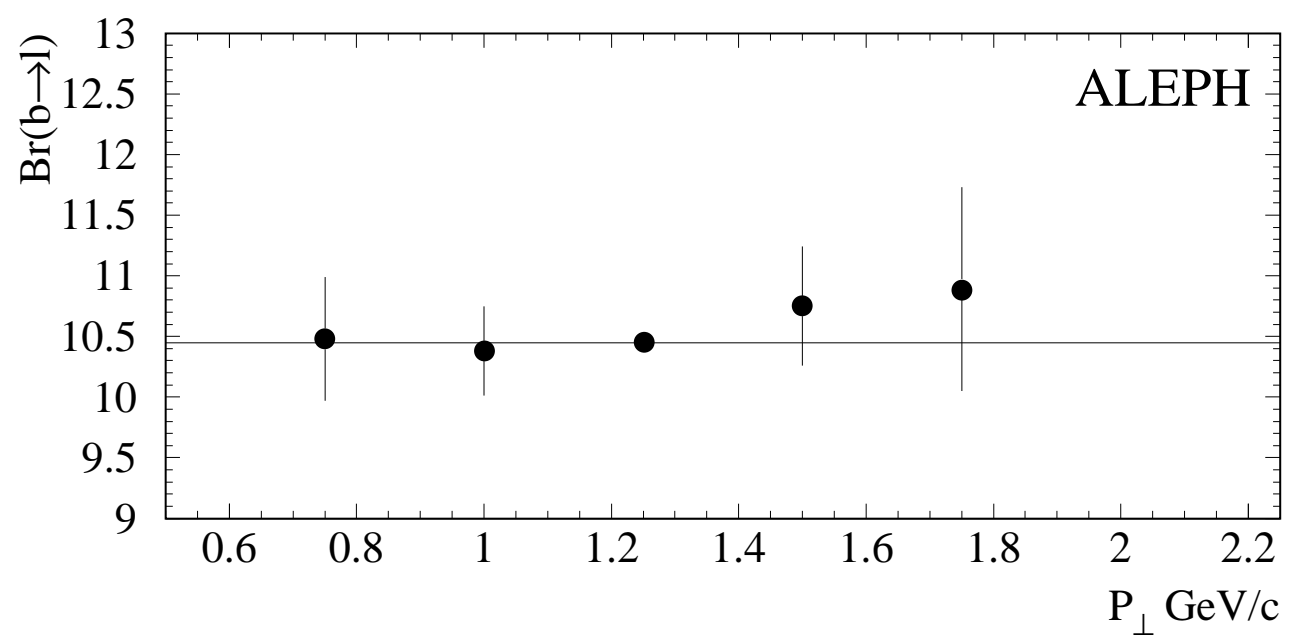

Figure 2: $\operatorname{Br}\left(b \rightarrow \ell^{-} \bar{\nu} \mathrm{X}\right)$ as a function of the $p_{\perp}$ cut. The error bars correspond to the statistical uncertainties which are uncorrelated to the uncertainty at a $p_{\perp}$ cut of $1.25 \mathrm{GeV} / \mathrm{c}$.

The magnitudes of the systematic uncertainties are given in table 5. The largest uncertainties are in the efficiencies and the modelling. Separating out the estimated systematic uncertainties from the shapes of the $b \rightarrow \ell, b \rightarrow c \rightarrow \ell$, and 
$c \rightarrow \ell$ spectra, the final result is:

$$
B r(b \rightarrow \ell \nu X)=0.1045 \pm 0.0043(\text { stat }) \pm 0.0036(\text { syst }) \pm 0.0047(\text { models })
$$

\begin{tabular}{|l|c|c|}
\hline Source & Variation & $\Delta \operatorname{Br}\left(b \rightarrow \ell^{-}\right)$ \\
\hline Monte Carlo statistics & $1 \sigma$ & 0.0016 \\
$R_{c} \cdot \operatorname{Br}\left(c \rightarrow \ell^{+}\right)$ & $10 \%$ & 0.0007 \\
$\operatorname{Br}\left(b \rightarrow c \rightarrow \ell^{+}\right)$ & $1 \sigma$ & 0.0001 \\
$\operatorname{Br}\left(b \rightarrow(\bar{c} s) \rightarrow \ell^{-}\right)$ & $50 \%$ & 0.0010 \\
$\operatorname{Br}\left(b \rightarrow \tau \rightarrow \ell^{-}\right)$ & $1 \sigma$ & 0.0004 \\
$\operatorname{Br}\left(b \rightarrow u \ell^{-}\right)$ & $50 \%$ & 0.0002 \\
$\varepsilon_{b}$ & $1 \sigma$ & $<0.0001$ \\
$\varepsilon_{c}$ & $1 \sigma$ & $<0.0001$ \\
lepton ID efficiency & $3 \%$ & 0.0021 \\
photon conversions & $10 \%$ & 0.0001 \\
electron background & $10 \%$ & 0.0001 \\
punch-through & $20 \%$ & 0.0005 \\
decaying hadrons & $10 \%$ & 0.0003 \\
gluon splitting & $100 \%$ & 0.0001 \\
Br $(\mathrm{B} \rightarrow \mathrm{J} / \psi)$ & $14 \%$ & 0.0007 \\
Eff $(\ell \ell)$ vs. Eff( $\ell)$ & $1.8 \%$ & 0.0018 \\
\hline \multicolumn{2}{|c|}{ TOTAL } \\
\hline
\end{tabular}

\begin{tabular}{|l|c|}
\hline Modelling uncertainties & $\Delta \operatorname{Br}\left(b \rightarrow \ell^{-}\right)$ \\
\hline$b \rightarrow \ell^{-}$model & 0.0045 \\
$c \rightarrow \ell^{+}$model & 0.0006 \\
$b \rightarrow c \rightarrow \ell^{+}$model & 0.0001 \\
$b \rightarrow(\bar{c} s) \rightarrow \ell^{-}$model & 0.0010 \\
$b$ fragmentation & 0.0008 \\
\hline \multicolumn{1}{|c|}{ TOTAL } & 0.0047 \\
\hline
\end{tabular}

Table 5: Estimated systematic uncertainties on $\operatorname{Br}\left(b \rightarrow \ell^{-} \bar{\nu} \mathrm{X}\right)$.

\subsection{Measurement of B- $\overline{\mathrm{B}}$ mixing}

As the lepton sign tags the particle-antiparticle nature of the decaying $b$ hadron, measurement of the proportion of opposite hemisphere dilepton events which have like sign yields information on the integrated mixing parameter $\chi$. This is defined 
as the probability that a produced $b$ state decays as a $\bar{b}$ state. It takes values from 0 to 0.5 . As only the neutral B mesons can mix,

$$
\chi=f_{d} \frac{B_{d}}{\langle B\rangle} \chi_{d}+f_{s} \frac{B_{s}}{\langle B\rangle} \chi_{s}
$$

where:

- $f_{i}$ are the fractions of mesons of type $i$.

- $B_{i}$ are the semileptonic branching fractions of mesons of type $i$.

- $\langle B\rangle$ is the average semileptonic branching fraction of $b$ hadrons.

Events are chosen which contain lepton candidates with $p_{\perp}>1.25 \mathrm{GeV} / \mathrm{c}$ in both hemispheres of the event defined in terms of the plane perpendicular to the thrust axis. If a hemisphere contains more than one lepton the one with the highest $p_{\perp}$ is used and the event counted once.

Dilepton candidates involving a fake lepton do not contribute equally to the like and unlike sign samples as some memory of the original quark charge remains. This background charge correlation has been measured from data from pairs of opposite hemisphere tracks chosen to satisfy only the kinematic criteria of the lepton selection. These yield

$$
\xi=\left(\frac{N_{\text {same charge }}}{N_{\text {pairs }}}\right)_{\text {background }}=0.48 \pm 0.01
$$

Given the low sensitivity of the measurement to this parameter (due to the low background contamination), any effect related to flavour or $p_{\perp}$ dependence can be effectively neglected with the present statistics.

The contributions to the like sign sample from the different lepton sources are given, in terms of $\chi$, in table 6 . The $f_{i j}$ give the dilepton sample composition and depend upon the lepton identification efficiency and the production and decay rates of the channels; they take into account any correlations between the hemispheres. They are obtained from Monte Carlo suitably weighted to use the most recent measurements of the various underlying parameters. In particular $\operatorname{Br}\left(b \rightarrow \ell^{-}\right), \operatorname{Br}\left(b \rightarrow c \rightarrow \ell^{+}\right)$, and fragmentation parameters are taken from the global fit analysis described in section 6 . Model dependence is examined by repeating the weighting for both the ACCMM and ISGW $W^{* *} b \rightarrow \ell$ decay models. Monte Carlo predictions for the major components of the dilepton sample in the ACCMM model are given in table 7 for various $p_{\perp}$ cuts.

Knowing the $f_{i j}$ and $\xi$, the integrated mixing parameter $\chi$ may be obtained from the proportion of pairs which have the same charge. 


\begin{tabular}{|c|c|c|c|c|}
\hline & $\begin{array}{c}b \rightarrow \ell \\
b \rightarrow \tau \rightarrow \ell \\
b \rightarrow \bar{c} \rightarrow \ell\end{array}$ & $b \rightarrow c \rightarrow \ell$ & $c \rightarrow \ell$ & fake \\
\hline $\begin{array}{c}b \rightarrow \ell \\
b \rightarrow \tau \rightarrow \ell \\
b \rightarrow \bar{c} \rightarrow \ell\end{array}$ & $\begin{array}{c}2 \chi(1-\chi) f_{11} \\
f_{11}=.785\end{array}$ & $\begin{array}{c}\left(\chi^{2}+(1-\chi)^{2}\right) f_{12} \\
f_{12}=.124\end{array}$ & - & $\begin{array}{c}f_{14} \xi \\
f_{14}=.064 \\
\end{array}$ \\
\hline$b \rightarrow c \rightarrow \ell$ & & $\begin{array}{c}2 \chi(1-\chi) f_{22} \\
f_{22}=.005\end{array}$ & - & $\begin{array}{l}f_{24}(1-\xi) \\
f_{24}=.006 \\
\end{array}$ \\
\hline$c \rightarrow \ell$ & & & $\begin{array}{c}0 \\
f_{33}=.007 \\
\end{array}$ & $\begin{array}{c}f_{34} \xi \\
f_{34}=.006 \\
\end{array}$ \\
\hline fake & & & & $\begin{array}{c}f_{44} \xi \\
f_{44}=.003\end{array}$ \\
\hline
\end{tabular}

Table 6: Contributions to the like sign fraction from the different channels. The $f_{i j}$ are given for the ACCMM model and $p_{\perp}>1.25 \mathrm{GeV} / \mathrm{c}$.

Results. An important check on the validity of the method comes from observation of the dependence of the final results on the $p_{\perp}$ cut. In table 8 the results for the two $b \rightarrow \ell$ decay models are shown for six values of this cut. It can be seen that above a cut of $1.0 \mathrm{GeV} / \mathrm{c}$ there is no trend in the values and the difference between the models is small and gets smaller as the cut value increases. For lower values of the $p_{\perp}$ cut, there is more overlap between the $b \rightarrow \ell$ spectrum and the $b \rightarrow c \rightarrow \ell$ spectrum and the sensitivity to the modelling becomes significant.

In figure 3 the results using the ACCMM model are plotted as a function of the $p_{\perp}$ cut with the uncorrelated statistical uncertainty of each point with respect to the $1.25 \mathrm{GeV} / \mathrm{c}$ point. No systematic trend is discernible.

A further check on the result may be made by evaluating it separately for $e e, \mu \mu$ and $e \mu$ pairs. No significant difference is observed. The values obtained are $\chi_{e e}=0.146 \pm 0.038, \chi_{\mu \mu}=0.088 \pm 0.024$, and $\chi_{e \mu}=0.110 \pm 0.022$.

No angular or momentum distributions are involved in the measurement of the mixing and therefore acceptance effects have little influence. The major contributor to the estimated systematic uncertainty results from unknowns concerning the cascade decay $b \rightarrow c \rightarrow \ell^{+}$. The rate and uncertainty are taken from the global analysis described in section 6 . The modelling and other systematic uncertainties are given in table 9 .

Separating out the estimated systematic uncertainties from the shapes of the 


\begin{tabular}{|c||c|c|c|c|c|}
\hline \multicolumn{1}{|c||}{\multirow{2}{*}{$\begin{array}{c}p_{\perp} \mathrm{cut} \\
\mathrm{GeV} / \mathrm{c}\end{array}$}} & \multicolumn{5}{c|}{ Monte Carlo composition: dileptons from $b$} \\
\cline { 2 - 6 } & $b \rightarrow \ell$ & $b \rightarrow \ell$ & $b \rightarrow \ell$ & $b \rightarrow \ell$ & $b \rightarrow \mathrm{X} \rightarrow \ell$ \\
\hline 0.75 & 56.4 & 2.6 & 19.0 & 2.1 & 3.3 \\
1.00 & 67.8 & 2.0 & 15.4 & 1.3 & 1.7 \\
1.25 & 76.0 & 1.8 & 12.1 & 0.7 & 0.8 \\
1.50 & 81.7 & 1.7 & 9.1 & 0.4 & 0.3 \\
1.75 & 84.8 & 1.7 & 7.5 & 0.2 & $<0.2$ \\
2.00 & 88.0 & 1.9 & 5.7 & 0.3 & $<0.3$ \\
\hline
\end{tabular}

\begin{tabular}{|c|c|c|c|c|}
\hline \multirow{3}{*}{$\begin{array}{l}p_{\perp} \mathrm{cut} \\
\mathrm{GeV} / \mathrm{c}\end{array}$} & \multicolumn{4}{|c|}{ Monte Carlo composition: other channels } \\
\hline & $c \rightarrow \ell$ & $b \rightarrow \ell$ & $b \rightarrow \mathrm{X} \rightarrow \ell$ & $\mathrm{X}$ \\
\hline & $c \rightarrow \ell$ & fake & fake & $\mathrm{X}$ \\
\hline 0.75 & 2.2 & 8.9 & 2.2 & 3.3 \\
\hline 1.00 & 1.4 & 7.3 & 1.2 & 1.9 \\
\hline 1.25 & 0.7 & 6.2 & 0.8 & 0.9 \\
\hline 1.50 & 0.3 & 5.2 & 0.4 & 0.9 \\
\hline 1.75 & $<0.2$ & 4.2 & 0.3 & 1.3 \\
\hline 2.00 & $<0.3$ & 3.6 & $<0.3$ & 0.5 \\
\hline
\end{tabular}

Table 7: Monte Carlo composition for various cuts (\%), using the ACCMM model. Here $b \rightarrow \mathrm{X} \rightarrow \ell$ groups together the three 'cascade' decay channels ( $\mathrm{X}=\tau, c$, $(\bar{c} s)$; the last column contains all the remaining spurious channels.

\begin{tabular}{|l||c|c|}
\hline \multirow{2}{*}{$p_{\perp}$ cut } & \multicolumn{2}{c|}{ Mixing with statistical uncertainty } \\
\cline { 2 - 3 } & ACCMM model & ISGW $^{* *}$ model \\
\hline 0.75 & $0.125 \pm 0.016$ & $0.137 \pm 0.015$ \\
1.00 & $0.106 \pm 0.014$ & $0.114 \pm 0.014$ \\
1.25 & $0.111 \pm 0.015$ & $0.115 \pm 0.015$ \\
1.50 & $0.105 \pm 0.017$ & $0.108 \pm 0.017$ \\
1.75 & $0.091 \pm 0.019$ & $0.093 \pm 0.019$ \\
2.00 & $0.118 \pm 0.028$ & $0.118 \pm 0.028$ \\
\hline
\end{tabular}

Table 8: Comparison between ACCMM and ISGW ${ }^{* *}$ decay model with full statistics. 


\begin{tabular}{|l|c|c|}
\hline Source & Variation & $\Delta \chi$ \\
\hline Monte Carlo statistics & $1 \sigma$ & 0.0052 \\
$R_{c} \cdot \operatorname{Br}\left(c \rightarrow \ell^{+}\right)$ & $10 \%$ & $<0.0001$ \\
$\operatorname{Br}\left(b \rightarrow c \rightarrow \ell^{+}\right)$ & $1 \sigma$ & 0.0053 \\
$\operatorname{Br}\left(b \rightarrow(\bar{c} s) \rightarrow \ell^{-}\right)$ & $50 \%$ & 0.0002 \\
$\operatorname{Br}\left(b \rightarrow \tau \rightarrow \ell^{-}\right)$ & $1 \sigma$ & 0.0002 \\
$\operatorname{Br}\left(b \rightarrow u \ell^{-}\right)$ & $50 \%$ & 0.0009 \\
$\varepsilon_{b}$ & $1 \sigma$ & 0.0001 \\
$\varepsilon_{c}$ & $1 \sigma$ & $<0.0001$ \\
lepton ID efficiency & $3 \%$ & $<0.0001$ \\
photon conversions & $10 \%$ & $<0.0001$ \\
electron background & $10 \%$ & $<0.0001$ \\
punch-through & $20 \%$ & 0.0005 \\
decaying hadrons & $10 \%$ & 0.0003 \\
gluon splitting & $100 \%$ & 0.0007 \\
$R_{b}$ & $5 \%$ & $<0.0001$ \\
Br $\left(b \rightarrow \ell^{-}\right)$ & $1 \sigma$ & 0.0022 \\
background charge correlation & $1 \sigma$ & 0.0006 \\
\hline \multicolumn{2}{|c|}{ TOTAL } & 0.0079 \\
\hline
\end{tabular}

\begin{tabular}{|c|c|}
\hline Modelling uncertainties & $\Delta \chi$ \\
\hline$b \rightarrow \ell$ model & 0.0020 \\
\hline$c \rightarrow \ell$ model & 0.0007 \\
\hline$b \rightarrow c \rightarrow \ell$ model & 0.0068 \\
\hline$b \rightarrow(\bar{c} s) \rightarrow \ell$ model & 0.0002 \\
\hline$b$ fragmentation & 0.0002 \\
\hline TOTAL & 0.0071 \\
\hline
\end{tabular}

Table 9: Estimated contributions to the systematic uncertainty on $\chi$. Modelling uncertainties are considered separately from the other sources. 


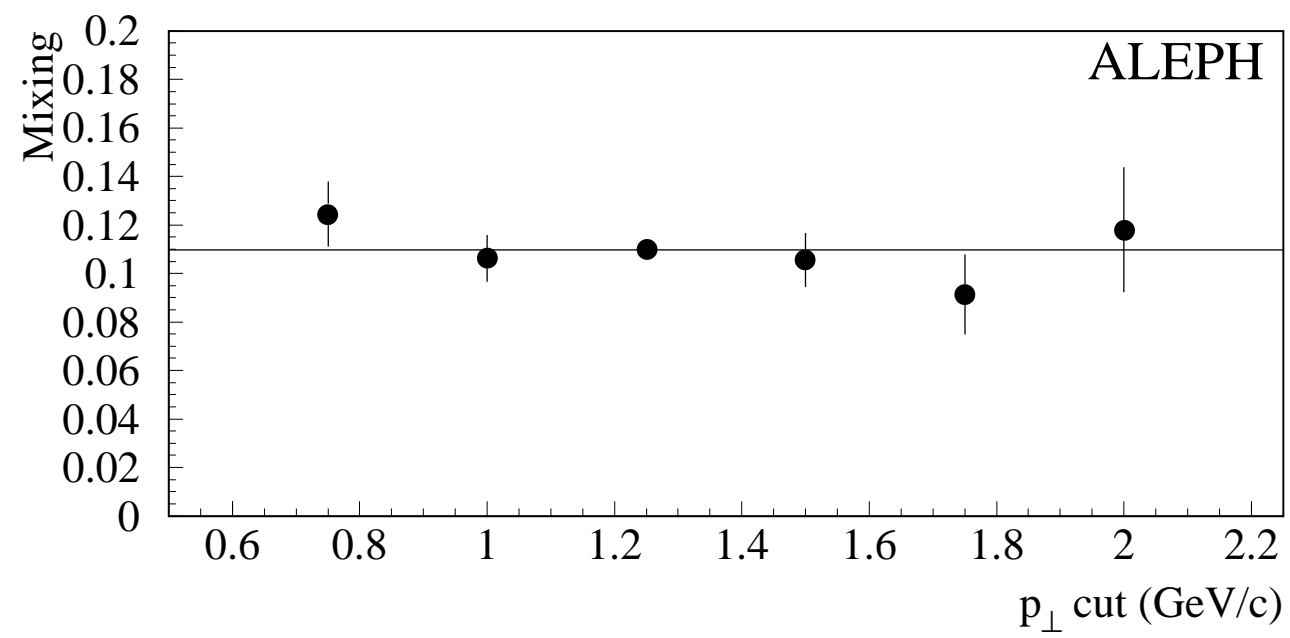

Figure 3: Stability of mixing measurement with respect to changing the $p_{\perp}$ cut.

$b \rightarrow \ell, b \rightarrow c \rightarrow \ell$, and $c \rightarrow \ell$ spectra the final result is:

$$
\chi=0.113 \pm 0.015(\text { stat }) \pm 0.008(\text { syst }) \pm 0.007 \text { (models) }
$$

\subsection{Measurement of $A_{F B}^{b}$}

The sign of the lepton reflects the nature of the decaying $b$ quark and hence the leptonic decays can be used to measure the forward-backward asymmetry for $b$ quark production in $\mathrm{Z}$ decay. This measurement is considerably more sensitive to the weak mixing angle $\sin ^{2} \theta_{W}$ than the corresponding lepton pair asymmetry.

Monte Carlo studies show that the event thrust axis, obtained using both charged and neutral particles, provides the best estimate of the $b \bar{b}$ axis from the $\mathrm{Z}$ decay, and so this is used to define the decay polar angle. Figure 4 shows that the angle between the original $b$ quark direction and the thrust axis is very small. Also shown are the angles between the $b$ quark and both the produced jet and the lepton. The jet containing the lepton is associated with that direction of the thrust axis with which it makes the smaller angle and then the polar angle for the $b$ quark is taken as the corresponding thrust angle if the lepton is negative or the reverse direction if the lepton is positive:

$$
\cos \theta_{b}=-Q \cos \theta_{\text {thrust }}
$$

where $Q$ is the lepton charge.

In events with more than one identified lepton, the one with the highest $p_{\perp}$ is used. 


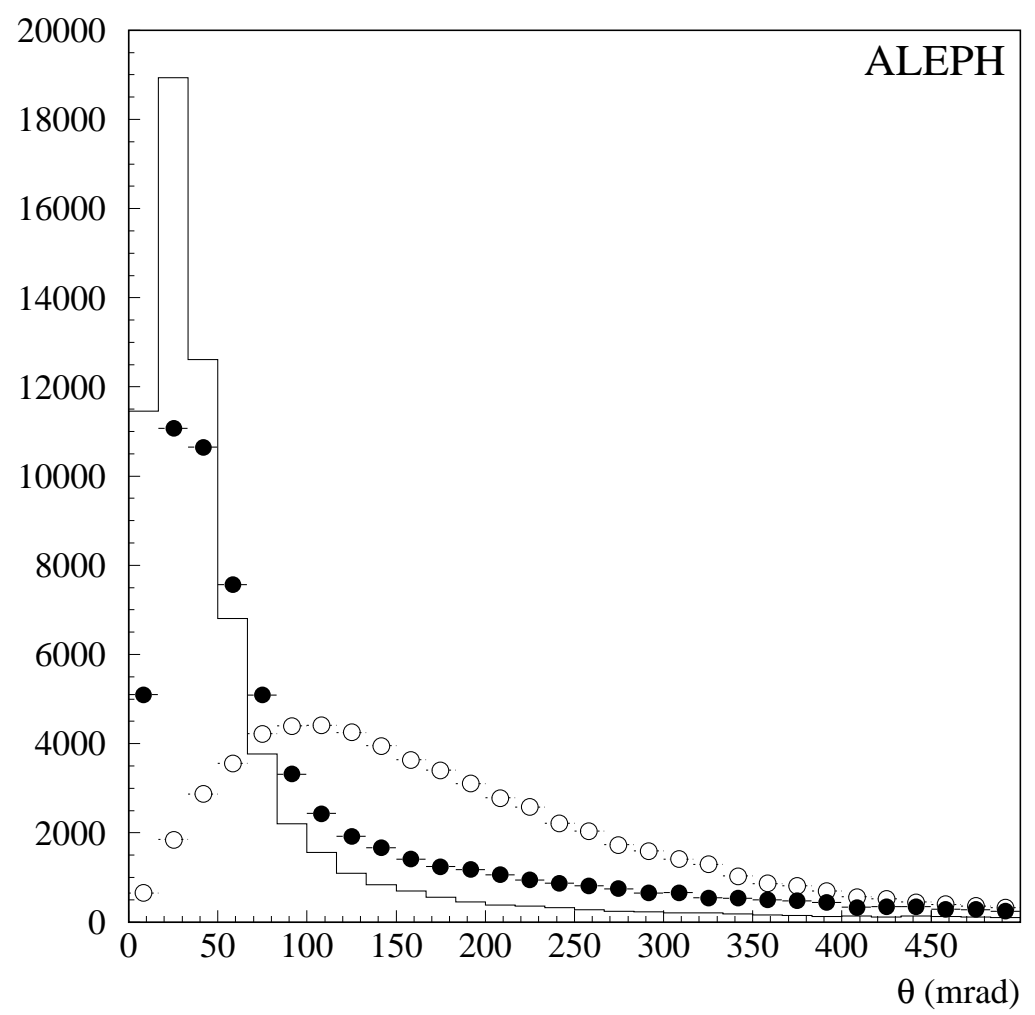

Figure 4: Angle between the initial $b$ quark direction and the thrust axis (solid line), the jet axis (solid circles) and the lepton (empty circles), for events with semileptonic $b$ decays. The vertical scale is arbitrary, and the plots have been normalized to equal areas. The jet axis is defined as in reference [1]. 
The uncorrected angular distributions for electrons and muons separately are shown in figures $5 a$ and $b$. These have to be corrected for acceptance effects due to the lack of uniform response over the complete polar angle range. The acceptance weights are normalized so that the total number of observed events is unchanged. The weights for the entire lepton sample are shown in figure 6 ; they have a small rise around $|\cos \theta|=0.6$ which is the region of the barrel/endcap overlap and a large rise for $|\cos \theta|>0.9$ due to losses close to the beam pipe. The latter region is excluded from the fit. The events are also weighted to take into account the variation as a function of $\left(p, p_{\perp}\right)$ and $\cos \theta_{\text {lepton }}$ of the lepton identification efficiency with respect to the Monte Carlo. In fact the applied weights have a negligible effect on the fitted asymmetry compared to the statistical uncertainty.
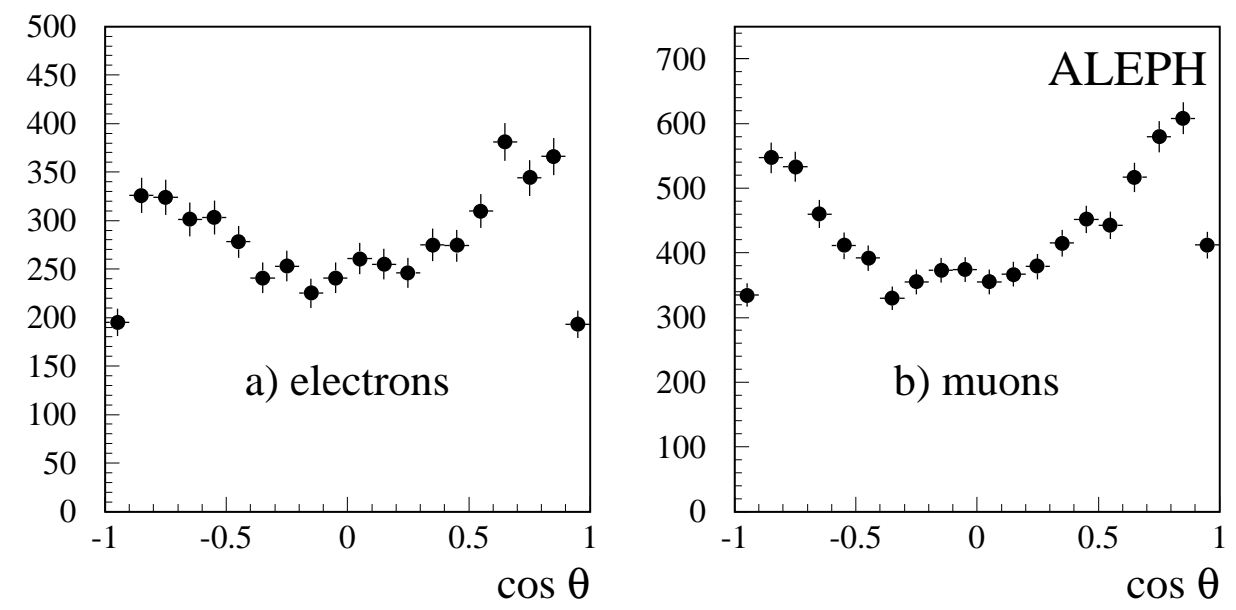

Figure 5: Observed angular distributions separately shown for the total electron $(a)$ and muon $(b)$ samples, at the peak energy.

The raw asymmetry, $A_{F B}^{o b s}$, is then obtained from an unbinned maximum likelihood fit of the weighted events to the form

$$
\frac{d \sigma}{d \cos \theta_{b}}=C\left(1+\cos ^{2} \theta_{b}+\frac{8}{3} A_{F B}^{o b s} \cos \theta_{b}\right)
$$

The acceptance corrected points for data at the peak energy are shown in figure 7 with the fitted curve; the final values for $A_{F B}^{o b s}$ at the seven energy points are given in the first column of table 10 .

Extraction of $A_{F B}^{b}$ The observed asymmetry, $A_{F B}^{o b s}$, must be corrected for dilution effects to find the true $b$ asymmetry at each energy point. Corrections arise from: 


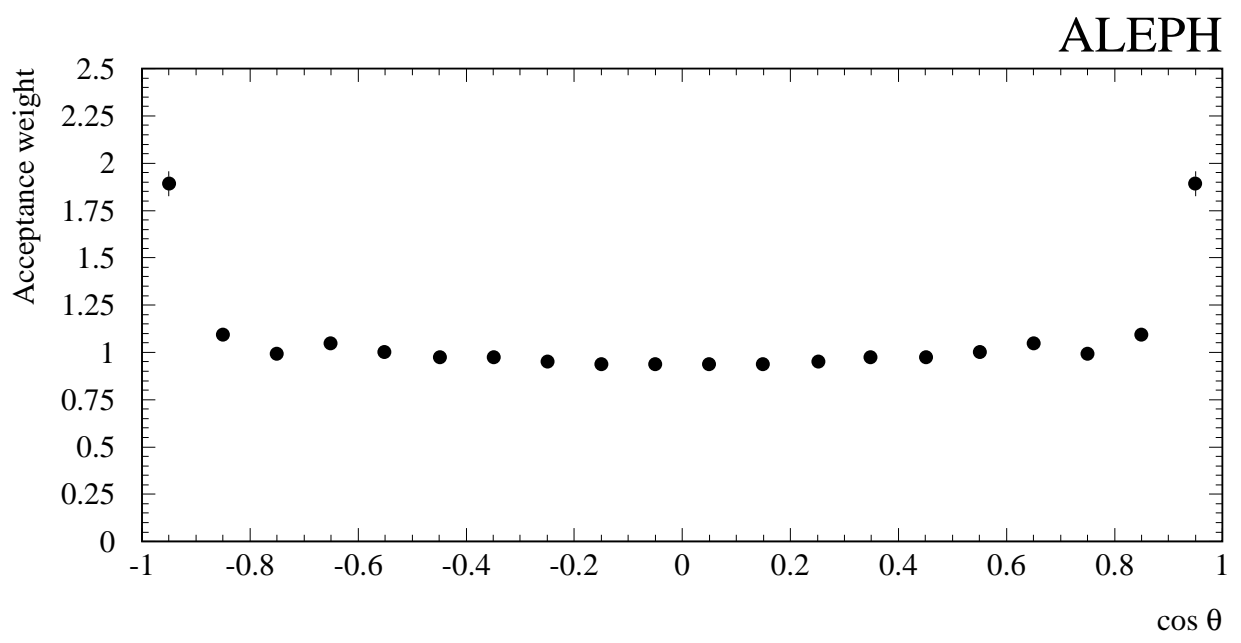

Figure 6: Acceptance weights determined from Monte Carlo.

\begin{tabular}{|l|r|r|r|}
\hline Energy Point & \multicolumn{1}{|c|}{$A_{F B}^{\text {obs }}$} & \multicolumn{1}{|c|}{$A_{F B}^{b}$} & No. of Z \\
\hline Peak $-3 \mathrm{GeV}$ & $0.036 \pm 0.063$ & $0.038 \pm 0.067 \pm 0.005$ & 6000 \\
Peak $-2 \mathrm{GeV}$ & $-0.009 \pm 0.047$ & $-0.017 \pm 0.076 \pm 0.003$ & 11500 \\
Peak $-1 \mathrm{GeV}$ & $0.028 \pm 0.033$ & $0.045 \pm 0.060 \pm 0.005$ & 21400 \\
Peak & $0.045 \pm 0.009$ & $0.081 \pm 0.016 \pm 0.005$ & 333600 \\
Peak $+1 \mathrm{GeV}$ & $0.041 \pm 0.029$ & $0.070 \pm 0.055 \pm 0.007$ & 29700 \\
Peak $+2 \mathrm{GeV}$ & $0.071 \pm 0.038$ & $0.121 \pm 0.069 \pm 0.011$ & 16800 \\
Peak $+3 \mathrm{GeV}$ & $0.085 \pm 0.045$ & $0.145 \pm 0.081 \pm 0.013$ & 12300 \\
\hline
\end{tabular}

Table 10: In the first column the values of the fitted raw asymmetry at seven energy points for the total sample are shown. The uncertainties are statistical only. In the second column the extracted asymmetry at seven energy points for the total sample is given. In the last column the number of $\mathrm{Z}$ collected at each point is shown. 


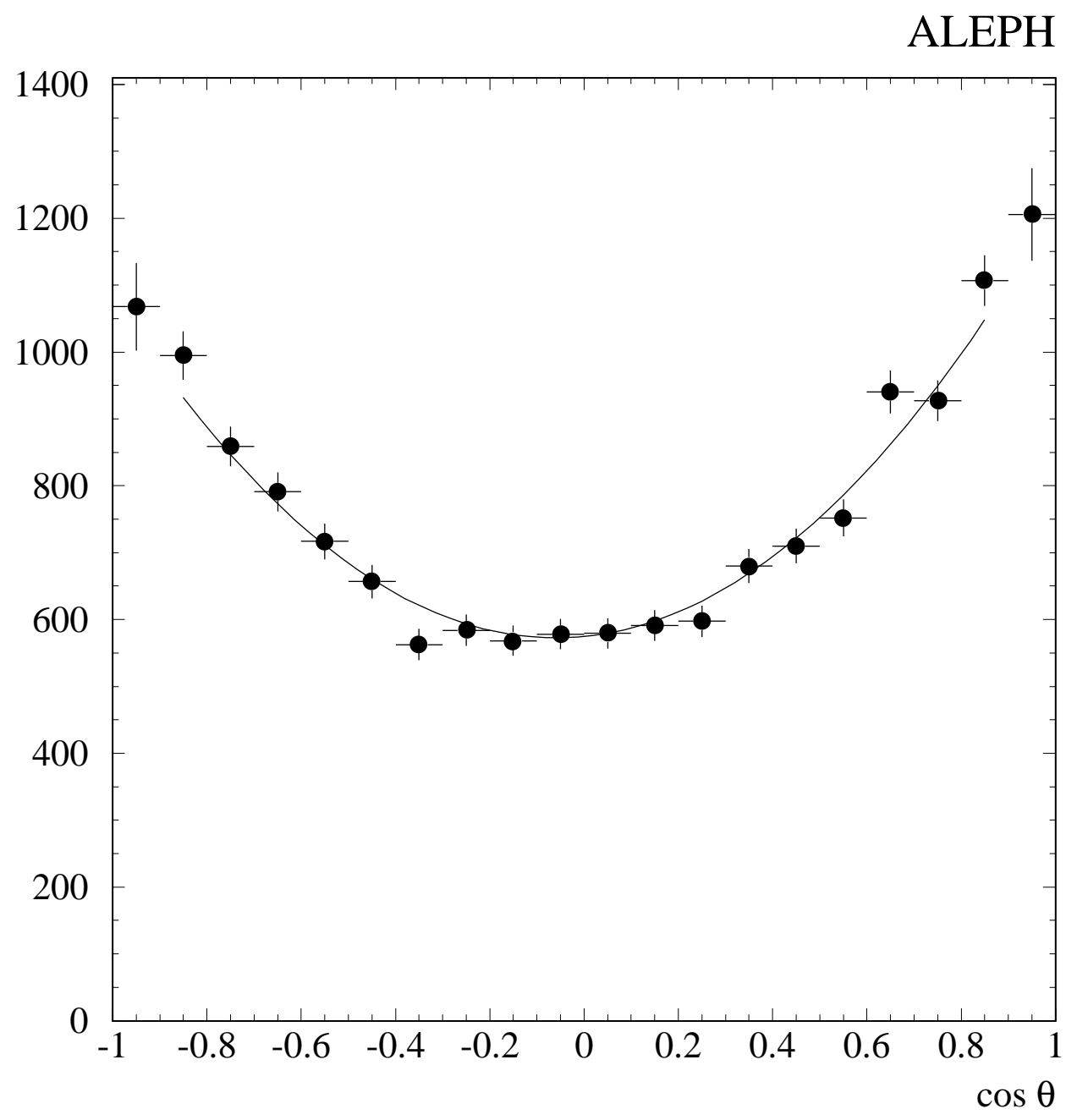

Figure 7: Acceptance corrected angular distribution, with the fitted curve superimposed. Peak energy only. 
- Leptons resulting from $b$ hadrons which have mixed and therefore have the wrong sign.

- Leptons resulting from the cascade decay $b \rightarrow c \rightarrow \ell^{+}$which yield the wrong charge and hence the reverse direction for the $b$ quark.

- Backgrounds from charm and light quark production in the selected sample.

The true $b$ asymmetry, $A_{F B}^{b}$, is obtained from

$$
A_{F B}^{b}=\frac{A_{F B}^{o b s}+\eta_{c \rightarrow \ell} A_{F B}^{c}-\eta_{b k g} A_{F B}^{b k g}}{(1-2 \chi)\left(\eta_{b \rightarrow \ell}+\eta_{b \rightarrow \tau \rightarrow \ell}+\eta_{b \rightarrow \bar{c} \rightarrow \ell}-\eta_{b \rightarrow c \rightarrow \ell}\right)}
$$

where the $\eta_{i}$ are the fractional contributions of process $i$ to the final sample composition and $A_{F B}^{b k g}$ is the asymmetry of the light quark contaminants.

For the small charm contribution the Standard Model is used to relate $A_{F B}^{c}$ to $A_{F B}^{b}$. The ratio

$$
\kappa=\frac{A_{F B}^{c}}{A_{F B}^{b}}
$$

is well predicted in the Standard Model and has almost no dependence on the top mass. The values of $\kappa$ used at the different centre of mass energies are taken from EXPOSTAR [23] and listed in table 11.

The $\eta_{i}$ are determined from the Monte Carlo simulation using $\operatorname{Br}\left(b \rightarrow \ell^{-}\right)$and $\operatorname{Br}\left(b \rightarrow c \rightarrow \ell^{+}\right)$from the global fit; the proportions have been given in table 1 . The use of different decay models for the $b \rightarrow \ell^{-}$spectrum has in practice no effect on the asymmetry and so only results from the ACCMM modelling are shown.

The background asymmetry resulting from non-prompt leptons and misidentified hadrons in the sample is also taken from the Monte Carlo. It is found to be

$$
A_{F B}^{b k g}=0.014 \pm 0.007
$$

and is nonzero due to residual leading particle effects which are preferentially selected by the lepton kinematic cuts.

Further corrections are required to obtain the Born level asymmetry at the peak, $A_{F B}^{0}(b)$, from which $\sin ^{2} \theta_{W}^{e f f}$ can be obtained. The only significant ones result from corrections for initial state photon radiation which decreases the effective centre of mass energy and final state gluon bremsstrahlung which decreases the asymmetry. They are discussed in section 7 .

Results. The stability of the extracted value of $A_{F B}^{b}$ as a function of the $p_{\perp}$ cut chosen is shown in figure 8 .

The uncertainties relate to the statistical differences of each point with respect to the standard cut at $1.25 \mathrm{GeV} / \mathrm{c}$. There is no evidence of any systematic dependence on the cut value. 


\begin{tabular}{|l|c|}
\hline Energy Point & $\kappa$ \\
\hline Peak $-3 \mathrm{GeV}$ & -5.56 \\
Peak $-2 \mathrm{GeV}$ & -1.49 \\
Peak $-1 \mathrm{GeV}$ & 0.10 \\
Peak & 0.77 \\
Peak $+1 \mathrm{GeV}$ & 0.91 \\
Peak $+2 \mathrm{GeV}$ & 1.10 \\
Peak $+3 \mathrm{GeV}$ & 1.21 \\
\hline
\end{tabular}

Table 11: Values of the ratio $\kappa$ at the various energy points, from EXPOSTAR.

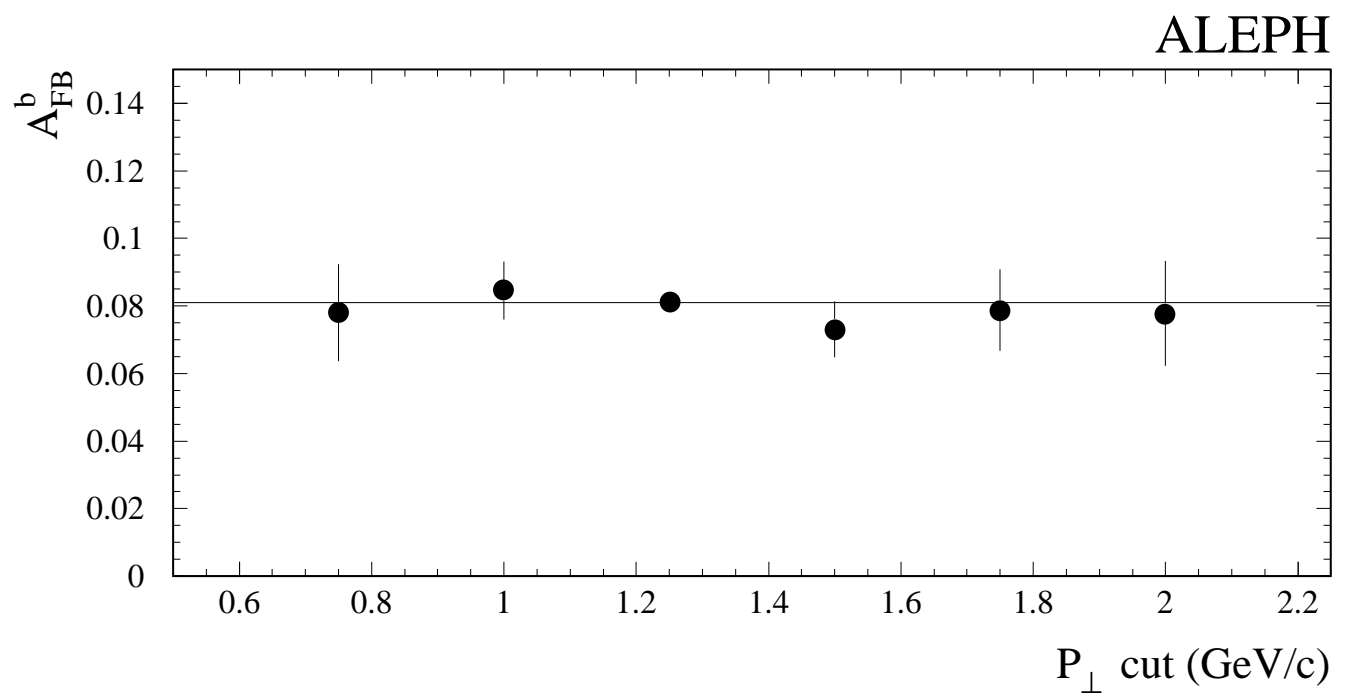

Figure 8: Extracted values of $A_{F B}^{b}$ at various $p_{\perp}$ cuts. The uncertainties are relative to the difference with respect to the value for the chosen cut. 


\begin{tabular}{|l|c|c|}
\hline Source & Variation & $\Delta A_{F B}^{b}$ \\
\hline Monte Carlo statistics & $1 \sigma$ & 0.0004 \\
$R_{c} \cdot \operatorname{Br}\left(c \rightarrow \ell^{+}\right)$ & $10 \%$ & 0.0006 \\
$\operatorname{Br}\left(b \rightarrow c \rightarrow \ell^{+}\right)$ & $1 \sigma$ & 0.0007 \\
$\operatorname{Br}\left(b \rightarrow(\bar{c} s) \rightarrow \ell^{-}\right)$ & $50 \%$ & $<0.0001$ \\
$\operatorname{Br}\left(b \rightarrow \tau \rightarrow \ell^{-}\right)$ & $1 \sigma$ & 0.0001 \\
$\operatorname{Br}\left(b \rightarrow u \ell^{-}\right)$ & $50 \%$ & 0.0001 \\
$\varepsilon_{b}$ & $1 \sigma$ & 0.0002 \\
$\varepsilon_{c}$ & $1 \sigma$ & 0.0010 \\
lepton ID efficiency & $3 \%$ & $<0.0001$ \\
photon conversions & $10 \%$ & $<0.0001$ \\
electron background & $10 \%$ & $<0.0001$ \\
punch-through & $20 \%$ & 0.0002 \\
decaying hadrons & $10 \%$ & 0.0001 \\
gluon splitting & $100 \%$ & 0.0003 \\
$R_{b}$ & $5 \%$ & 0.0009 \\
Br(b $\left.\rightarrow \ell^{-}\right)$ & $1 \sigma$ & 0.0002 \\
$\chi$ & see text & 0.0037 \\
$A_{F B}^{\text {background }}$ & $1 \sigma$ & 0.0020 \\
\hline \multicolumn{2}{|c|}{ TOTAL } & 0.0046 \\
\hline
\end{tabular}

\begin{tabular}{|l|c|}
\hline Modelling uncertainties & $\Delta A_{F B}^{b}$ \\
\hline$b \rightarrow \ell^{-}$model & 0.0003 \\
$b \rightarrow c \rightarrow \ell^{+}$model & 0.0007 \\
$b \rightarrow(\bar{c} s) \rightarrow \ell^{-}$model & $<0.0001$ \\
$c \rightarrow \ell^{+}$model & 0.0001 \\
$b$ fragmentation & 0.0004 \\
\hline \multicolumn{1}{|c|}{ TOTAL } & 0.0009 \\
\hline
\end{tabular}

Table 12: Estimated contributions to the systematic uncertainty on $A_{F B}^{b}$ at the Z peak. Modelling uncertainties are considered separately from the other sources. 
The AlEPH detector is symmetric with respect to the polar angle. Detector inhomogeneities can only cause a systematic problem for the asymmetry measurement if they are, at the same time, both forward-backward and charge asymmetric. Examination of dimuon and Bhabha pairs reveal no such correlated asymmetry within the apparatus. Simulations also show that the use of the thrust axis to approximate the quark direction produces a negligible effect on the asymmetry.

The estimated systematic error in the measurement of $A_{F B}^{b}$ is dominated by the corrections which must be applied to $A_{F B}^{o b s}$. The most significant arises from the mixing correction, for which $\chi$ is taken from the previous analysis. However, when calculating the contribution from $\chi$ to the systematic error, allowance is made for the contributions to $\delta \chi$ resulting from uncertainties which are explicitly considered for the asymmetry.

The next most significant contribution arises from the background asymmetry whose uncertainty is given by the statistical error on the Monte Carlo sample. It is considerably smaller than the mixing uncertainty. Other contributions to the systematic error are listed in table 12 .

After all corrections the value extracted for $A_{F B}^{b}$ at peak $(\sqrt{s}=91.24 \mathrm{GeV})$ is

$$
A_{F B}^{b}=0.081 \pm 0.016(\text { stat }) \pm 0.005 \text { (syst) } \pm 0.001 \text { (models) }
$$

and the energy dependence is plotted in figure 9. The statistical uncertainty is totally dominant. The main contribution to the systematic uncertainty, mixing, will also decrease with additional data.

\section{Global analysis}

Basic principles. In the global analysis all the quantities discussed in the previous section are obtained from a simultaneous fit to the $p$ and $p_{\perp}$ spectra of both single and dilepton events. This also allows a measurement of the fragmentation within the framework of a particular model and, as the fit covers the full range of transverse momentum, analogous quantities for the charm sector are obtained. A major advantage of this approach is that it also gives the statistical correlations between the measured quantities.

Three samples of events $\left(\mathcal{N}, \mathcal{D}^{\Longrightarrow}\right.$, and $\left.\mathcal{D}^{\longleftrightarrow}\right)$ are considered for the fit, as in section 5.2 , but the cut on $p_{\perp}$ is removed. The dilepton samples are also split into same charge $\mathcal{D}_{\text {s.c. }}$ and opposite charge $\mathcal{D}_{\text {o.c. }}$.

The different processes which contribute to the lepton and dilepton samples have different $p$ and $p_{\perp}$ spectra which allow them to be separated. The shape of the lepton spectra for each process is described in section 3.2.

- Primary $b$ decays dominate the high $\left(p, p_{\perp}\right)$ region for both $\mathcal{N}$ and $\mathcal{D}^{\longleftrightarrow}$. Such events effectively determine $R_{b}, \operatorname{Br}\left(b \rightarrow \ell^{-}\right),\left\langle x_{b}\right\rangle$ and $A_{F B}^{b}$, whilst $\mathcal{D}_{\text {s.c. }}^{\longleftrightarrow}$ determines $\chi$. 


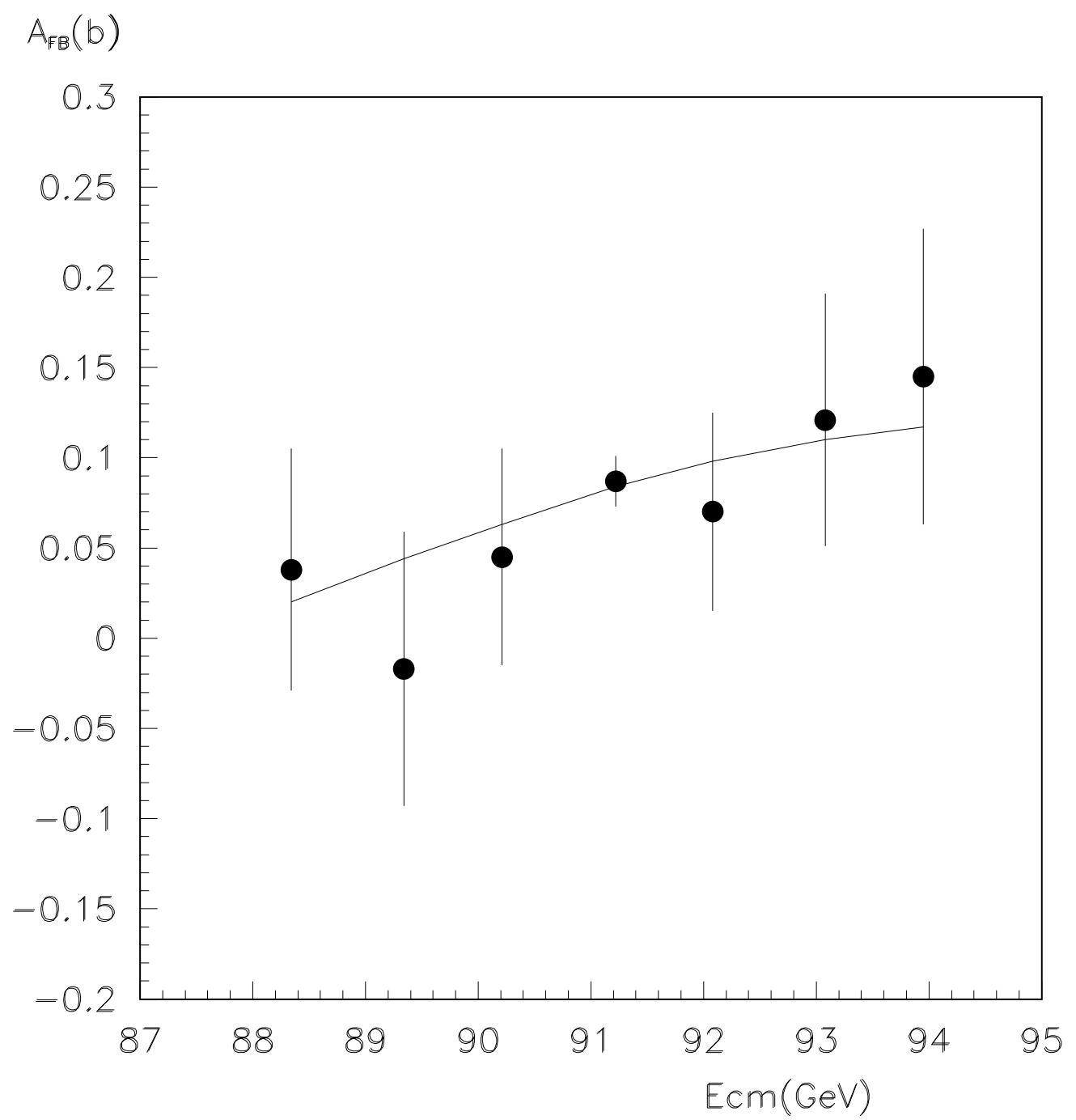

Figure 9: Extracted values of $A_{F B}^{b}$ as a function of the centre-of-mass energy. The central point is taken from the global fit. The curve is the result of a fit to the Standard Model (see section 7). 
- Cascade $b$ decays have softer spectra for both $p$ and $p_{\perp}$. Events with one of the leptons from a cascade decay dominate $\mathcal{D}_{\text {o.c. }}^{\Rightarrow}$ and yield a measurement of $\operatorname{Br}\left(b \rightarrow c \rightarrow \ell^{+}\right)$.

- Leptons from charm decay populate the low $p_{\perp}$ region of $\mathcal{N}$ and $\mathcal{D}_{\text {o.c. }}^{\longleftrightarrow}$ and enable measurements of $R_{c}, A_{F B}^{c}$, and $\left\langle x_{c}\right\rangle$. In principle one could also determine $\operatorname{Br}\left(c \rightarrow \ell^{+}\right)$from the low $p_{\perp}$ dileptons, but the overlap with the cascade decays makes separation difficult with present statistics.

- The rates $\operatorname{Br}\left(b \rightarrow \tau \rightarrow \ell^{-}\right), \operatorname{Br}\left(b \rightarrow(\bar{c} s) \rightarrow \ell^{-}\right)$, and $\operatorname{Br}\left(c \rightarrow \ell^{+}\right)$are taken from the best measurements available as described in section 4.1.

- The $\left(p, p_{\perp}\right)$ distributions of the fake sample are taken from Monte Carlo simulation, after corrections for lepton identification efficiencies and contaminations as described in reference [1].

Choice of kinematic variables. For each lepton pair there are essentially four kinematic quantities, $p_{\| i}, p_{\perp i}(i=1,2)$ with $p_{\|}$and $p_{\perp}$ being the longitudinal and transverse components of the lepton momentum with respect to the jet axis. Combinations of these were examined using a Fisher test method to maximize discrimination of the $\left(b \rightarrow \ell^{-}\right)\left(\bar{b} \rightarrow \ell^{+}\right)$component from the others. The best variable was found to be

$$
P_{\otimes}=p_{\perp 1} p_{\| 2}+p_{\perp 2} p_{\| 1}
$$

similar to the one originally proposed by Mark II [24]. A second variable $P_{\perp m}=$ $\operatorname{Min}\left(p_{\perp 1}, p_{\perp 2}\right)$ is chosen because of its good discriminating power and its limited correlation with $P_{\otimes}$.

These variables are also effective for the $\mathcal{D}_{\text {o.c. }}^{\Rightarrow}$ dilepton component where the signal events result from $b \rightarrow\left(c \ell^{-}\right) ; c \rightarrow \ell^{+} \mathrm{X}$. Dilepton decays of the $\mathrm{J} / \psi$ are a major contaminant but these two processes populate different areas of the $\left(P_{\otimes}, P_{\perp m}\right)$ plane and so the analysis becomes insensitive to uncertainties in the $\mathrm{B} \rightarrow \mathrm{J} / \psi+\mathrm{X}$ branching ratio.

Analysis procedure. Leptons are analysed in the $\left(p, p_{\perp},-Q \cos \theta\right)$ space while both sets of dileptons are analysed in the $\left(P_{\otimes}, P_{\perp m}\right)$ plane. The definition of $-Q \cos \theta$ is the same as in section 5.4. Results are obtained from a binned maximum likelihood fit of the weighted Monte Carlo assuming Poissonian fluctuations. The likelihood is the sum of three components from $\mathcal{N}, \mathcal{D}^{\longleftrightarrow}$ and $\mathcal{D} \Longrightarrow$; the likelihood function is given in the appendix. In the fit, only the fragmentation parameters $\left\langle x_{b}\right\rangle$ and $\left\langle x_{c}\right\rangle$ distort the $\left(p, p_{\perp}\right)$ spectra; all the other parameters appear as simple multiplicative numbers for the various components.

All lepton candidates with $p>3 \mathrm{GeV} / \mathrm{c}$ are used for all measurements except as follows: 
- As $A_{F B}^{b}$ is energy dependent, the distribution in $-Q \cos \theta$ was only considered at the peak energy of $91.24 \mathrm{GeV}$.

- The dilepton charge information was only used for the mixing measurement when both leptons had $p_{\perp}>1.0 \mathrm{GeV} / \mathrm{c}$. As was demonstrated in section 5.3, use of the ISGW $W^{* *} b \rightarrow \ell^{-}$decay model rather than ACCMM starts to have a significant effect as the $p_{\perp}$ region is extended to lower values. This is because the softer spectrum reduces the $b \rightarrow c \rightarrow \ell^{+}$component which is the principal background source. The cut at $1.0 \mathrm{GeV} / \mathrm{c}$ provides the most accurate value for $\chi$ from the global analysis when both statistical and systematic uncertainties are taken into account.

Results. In table 13 the results of the fit for the two decay models, ISGW ${ }^{* *}$ and ACCMM are given. It can be seen that the softer ISGW** spectrum leads to a $2 \%$ increase in the value of $R_{b}$ and a harder fragmentation function. The procedures adopted to estimate the $b \rightarrow \ell^{-}, b \rightarrow c \rightarrow \ell^{+}$, and $c \rightarrow \ell^{+}$modelling uncertainties are described in section 3.2 .

\begin{tabular}{|c|c|c|c|}
\hline Parameter & $\begin{array}{c}\text { ACCMM } \\
\text { Spectrum }\end{array}$ & $\begin{array}{c}\text { ISGW }^{* *} \\
\text { Spectrum }\end{array}$ & $\begin{array}{c}\text { Statistical } \\
\text { Uncertainty }\end{array}$ \\
\hline \hline$R_{b}$ & 0.2162 & 0.2215 & 0.0062 \\
$R_{c}$ & 0.1670 & 0.1621 & 0.0054 \\
\hline \hline $\operatorname{Br}\left(b \rightarrow \ell^{-}\right)$ & 0.1120 & 0.1159 & 0.0033 \\
$\mathrm{Br}\left(b \rightarrow c \rightarrow \ell^{+}\right)$ & 0.0881 & 0.0756 & 0.0025 \\
\hline \hline$<x_{b}>$ & 0.7037 & 0.7245 & 0.0035 \\
$<x_{c}>$ & 0.4883 & 0.4865 & 0.0083 \\
\hline \hline$\chi$ & 0.109 & 0.118 & 0.014 \\
\hline \hline$A_{F B}^{b}$ & 0.086 & 0.088 & 0.014 \\
\hline \hline$A_{F B}^{c}$ & 0.091 & 0.106 & 0.020 \\
\hline
\end{tabular}

Table 13: Global analysis: effect of the semileptonic primary $b$ decay modelling

Other systematic uncertainties arise from experimental uncertainties associated with lepton identification and input branching ratios not obtained from the fit. Their effect on the measured parameters are given in tables 14, 15 and 16 .

The final results are given in table 17 and the statistical correlation matrix from the fit in table 18 . The full correlation matrix, including statistical, systematic and modelling errors is given in table 19 .

It should be noted that: 


\begin{tabular}{|c|c|c|c|c|c|c|c|}
\hline Par. & $\begin{array}{c}\mathrm{e} \\
\text { eff. }\end{array}$ & $\begin{array}{c}\mu \\
\text { eff. }\end{array}$ & $\begin{array}{c}\gamma \\
\text { conv. }\end{array}$ & $\begin{array}{c}\mathrm{e} \\
\text { mis. }\end{array}$ & $\begin{array}{c}\pi / \mathrm{K} \\
\text { decay }\end{array}$ & $\begin{array}{c}\text { punch } \\
\text { through }\end{array}$ & $A_{\text {charge }}^{\text {back. }}$ \\
\hline \hline$R_{b}$ & 0.02 & 0.02 & 0.06 & 0.02 & 0.16 & 0.28 & \\
$R_{c}$ & 0.47 & 0.40 & 0.62 & 0.24 & 0.57 & 1.03 & \\
$\operatorname{Br}\left(b \rightarrow \ell^{-}\right)$ & 0.18 & 0.15 & 0.13 & 0.08 & 0.04 & 0.16 & \\
$\operatorname{Br}\left(b \rightarrow c \rightarrow \ell^{+}\right)$ & 0.15 & 0.18 & 0.06 & 0.01 & 0.15 & 0.28 & \\
$<x_{b}>$ & 0.1 & 0.1 & $<0.1$ & $<0.1$ & $<0.1$ & 0.1 & \\
$<x_{c}>$ & 0.3 & 0.1 & 0.1 & $<0.1$ & 0.1 & 0.1 & \\
$\chi$ & 0.02 & 0.04 & 0.03 & $<0.01$ & 0.12 & 0.21 & 0.05 \\
$A_{F B}^{b}$ & 0.05 & 0.02 & 0.03 & $<0.01$ & 0.08 & $<0.01$ & \\
$A_{F B}^{c}$ & 0.16 & 0.07 & 0.31 & 0.11 & 0.37 & 0.42 & \\
\hline
\end{tabular}

Table 14: Global analysis: experimental systematic uncertainties (units of $10^{-2}$ ).

\begin{tabular}{|c|c|c|c|c|c|c|}
\hline Par. & $c \rightarrow \ell^{+}$ & $b \rightarrow \tau \rightarrow \ell^{-}$ & $b \rightarrow(\bar{c} s) \rightarrow \ell^{-}$ & $\mathrm{B} \rightarrow \mathrm{J} / \psi$ & $b \rightarrow u$ & $A_{F B}^{\text {bkg }}$ \\
\hline \hline$R_{b}$ & 0.10 & 0.03 & 0.06 & 0.03 & 0.22 & \\
$R_{c}$ & 0.93 & 0.06 & 0.50 & 0.06 & 0.06 & \\
$\operatorname{Br}\left(b \rightarrow \ell^{-}\right)$ & 0.05 & 0.03 & 0.03 & 0.02 & 0.04 & \\
$\operatorname{Br}\left(b \rightarrow c \rightarrow \ell^{+}\right)$ & 0.02 & 0.09 & 0.20 & 0.06 & 0.41 & \\
$<x_{b}>$ & $<0.1$ & 0.1 & 0.1 & 0.2 & 0.4 & \\
$<x_{c}>$ & $<0.1$ & 0.1 & 0.3 & 0.1 & 0.3 & \\
$\chi$ & $<0.01$ & 0.07 & 0.20 & 0.04 & 0.16 & \\
$A_{F B}^{o b s}$ & $<0.01$ & 0.01 & 0.04 & 0.02 & 0.02 & 0.07 \\
$A_{F B}^{b}$ & $<0.01$ & 0.03 & 0.09 & 0.02 & $<0.01$ & 0.07 \\
$A_{F B}^{c}$ & 0.04 & 0.11 & 0.47 & 0.02 & 0.15 & 1.38 \\
\hline
\end{tabular}

Table 15: Global analysis: systematic uncertainties from branching ratios (units of $\left.10^{-2}\right)$. 


\begin{tabular}{|c|c|c|c|}
\hline Par. & $c \rightarrow \ell^{+}$ & $b \rightarrow c \rightarrow \ell^{+}$ & $b \rightarrow \ell^{-}$ \\
\hline \hline$R_{b}$ & 0.09 & 0.04 & 0.26 \\
$R_{c}$ & 0.40 & 0.54 & 0.25 \\
$\operatorname{Br}\left(b \rightarrow \ell^{-}\right)$ & 0.09 & 0.14 & 0.20 \\
$\operatorname{Br}\left(b \rightarrow c \rightarrow \ell^{+}\right)$ & 0.03 & 0.79 & 0.62 \\
$<x_{b}>$ & 0.1 & 0.1 & 1.0 \\
$<x_{c}>$ & 0.5 & 0.4 & 0.1 \\
$\chi$ & 0.04 & 0.58 & 0.43 \\
$A_{F B}^{b}$ & 0.03 & 0.02 & 0.10 \\
$A_{F B}^{c}$ & 0.11 & 0.14 & 0.72 \\
\hline
\end{tabular}

Table 16: Global analysis: systematic uncertainties from decay models (units of $\left.10^{-2}\right)$.

\begin{tabular}{|c|c|c|c|c|c|}
\hline Parameter & Value & $\begin{array}{c}\text { Statistical } \\
\text { Uncertainty }\end{array}$ & $\begin{array}{c}\text { Systematic } \\
\text { Uncertainty }\end{array}$ & $\begin{array}{c}\text { Model } \\
\text { Uncertainty }\end{array}$ & $\begin{array}{c}\text { Total } \\
\text { Uncertainty }\end{array}$ \\
\hline \hline$R_{b}$ & 0.2188 & 0.0062 & 0.0041 & 0.0028 & 0.0079 \\
$R_{c}$ & 0.1646 & 0.0054 & 0.0182 & 0.0072 & 0.0203 \\
\hline \hline $\mathrm{Br}\left(b \rightarrow \ell^{-}\right)$ & 0.1139 & 0.0033 & 0.0033 & 0.0026 & 0.0053 \\
$\mathrm{Br}\left(b \rightarrow c \rightarrow \ell^{+}\right)$ & 0.0819 & 0.0025 & 0.0061 & 0.0100 & 0.0120 \\
\hline \hline$<x_{b}>$ & 0.714 & 0.004 & 0.005 & 0.010 & 0.0012 \\
$<x_{c}>$ & 0.487 & 0.008 & 0.006 & 0.006 & 0.0012 \\
\hline \hline$\chi$ & 0.114 & 0.014 & 0.004 & 0.007 & 0.016 \\
\hline \hline$A_{F B}^{b}$ & 0.087 & 0.014 & 0.002 & 0.001 & 0.014 \\
\hline$A_{F B}^{c}$ & 0.099 & 0.020 & 0.016 & 0.007 & 0.027 \\
\hline
\end{tabular}

Table 17: Global analysis: final results. 


\begin{tabular}{|c|c|c|c|c|c|c|c|c|}
\hline$\rho$ & $R_{c}$ & $<x_{b}>$ & $<x_{c}>$ & $\begin{array}{c}\mathrm{Br} \\
(b \rightarrow \ell)\end{array}$ & $\begin{array}{c}\mathrm{Br} \\
(b \rightarrow c \rightarrow \ell)\end{array}$ & $\chi$ & $A_{F B}^{b}$ & $A_{F B}^{c}$ \\
\hline$R_{b}$ & -0.48 & 0.23 & -0.05 & -0.94 & -0.38 & -0.07 & 0.00 & 0.05 \\
$R_{c}$ & & 0.06 & 0.49 & 0.47 & -0.31 & 0.09 & -0.01 & -0.07 \\
$<x_{b}>$ & & & 0.12 & -0.35 & -0.27 & -0.01 & 0.00 & 0.00 \\
$<x_{c}>$ & & & & 0.15 & -0.29 & 0.05 & -0.04 & 0.00 \\
$\operatorname{Br}(b \rightarrow \ell)$ & & & & & 0.25 & 0.09 & 0.00 & -0.04 \\
$\operatorname{Br}(b \rightarrow c \rightarrow \ell)$ & & & & & & -0.07 & 0.00 & -0.01 \\
$\chi$ & & & & & & & 0.21 & 0.00 \\
$A_{F B}^{b}$ & & & & & & & & 0.21 \\
\hline
\end{tabular}

Table 18: Global analysis: statistical correlation matrix.

\begin{tabular}{|c|c|c|c|c|c|c|c|c|}
\hline$\rho$ & $R_{c}$ & $\left\langle x_{b}\right\rangle$ & $\left.<x_{c}\right\rangle$ & $\begin{array}{c}\mathrm{Br} \\
(b \rightarrow \ell)\end{array}$ & $\begin{array}{c}\mathrm{Br} \\
(b \rightarrow c \rightarrow \ell)\end{array}$ & $\chi$ & $A_{F B}^{b}$ & $A_{F B}^{c}$ \\
\hline$R_{b}$ & 0.08 & 0.35 & -0.16 & -0.55 & -0.21 & -0.01 & 0.00 & 0.08 \\
$R_{c}$ & & -0.12 & -0.24 & 0.04 & 0.13 & -0.19 & -0.05 & -0.20 \\
$<x_{b}>$ & & & -0.11 & 0.24 & -0.65 & 0.24 & 0.07 & 0.32 \\
$<x_{c}>$ & & & & 0.05 & 0.21 & 0.14 & -0.01 & 0.00 \\
$\operatorname{Br}(b \rightarrow \ell)$ & & & & & 0.05 & 0.25 & 0.02 & 0.10 \\
$\operatorname{Br}(b \rightarrow c \rightarrow \ell)$ & & & & & & 0.02 & -0.07 & -0.29 \\
$\chi$ & & & & & & & 0.20 & 0.12 \\
$A_{F B}^{b}$ & & & & & & & & 0.22 \\
\hline
\end{tabular}

Table 19: Global analysis: full correlation matrix 
- As many parameters are fitted, uncertainties which in the high $p_{\perp}$ analyses are introduced as systematic naturally appear here as statistical. This is particularly true for the contribution from the mixing uncertainty for $A_{F B}^{b}$.

- The ratios $R_{b}$ and $\operatorname{Br}\left(b \rightarrow \ell^{-}\right)$have a high negative statistical correlation; this is because the product is well measured (better than $1 \%$ statistically) from the lepton sample. Systematic uncertainties from lepton identification only significantly affect the branching ratio measurement as demonstrated with the high $p_{\perp}$ analysis of section 5.2 and this reduces the overall correlation.

- Modelling uncertainties dominate the errors in the measurement of the $b$ fragmentation parameter and the $\operatorname{Br}\left(b \rightarrow c \rightarrow \ell^{+}\right)$.

- The charm fragmentation parameter agrees well with the ALEPH measurement based on $\mathrm{D}^{*}$ production [25].

- When a comparison can be done between the global and high $p_{\perp}$ analysis, the results are in good agreement, after due allowance for the overlap of data samples and the correlation of systematic uncertainties.

- For the values of the QCD parameters used in HVFL [5], the fragmentation measurements correspond to values of the Peterson parameter of $\varepsilon_{b}=0.0032 \pm 0.0017$ and $\varepsilon_{c}=0.066 \pm 0.014$ for $b$ and $c$ quarks respectively.

The results of the fit are displayed on the data distributions in figures 10 and 11 , with the predicted components shown.

Consistency check. To check for consistency between the electron and muon samples the fit is repeated on each sample independently, fitting for $\operatorname{Br}\left(b \rightarrow \ell^{-}\right)$, $\operatorname{Br}\left(b \rightarrow c \rightarrow \ell^{+}\right), \operatorname{Br}\left(c \rightarrow \ell^{+}\right),\left\langle x_{b}>\right.$ and $\left\langle x_{c}>\right.$ with the values of $R_{b}$ and $R_{c}$ taken from the Standard Model. The fraction of the dilepton sample containing one electron and one muon is not used here. The results are given in table 20 with statistical uncertainties only. The agreement between the electron and muon results suggests the backgrounds in the two cases are well estimated. The value obtained for $\operatorname{Br}\left(c \rightarrow \ell^{+}\right)$is also consistent with the world average value used in the full analysis.

\section{$7 \quad$ Extraction of $\sin ^{2} \theta_{W}^{e f f}$}

The tree level forward-backward $b$ and $c$ asymmetries are related to the ratio of the vector and axial vector coupling constants, and, within the Standard Model, 

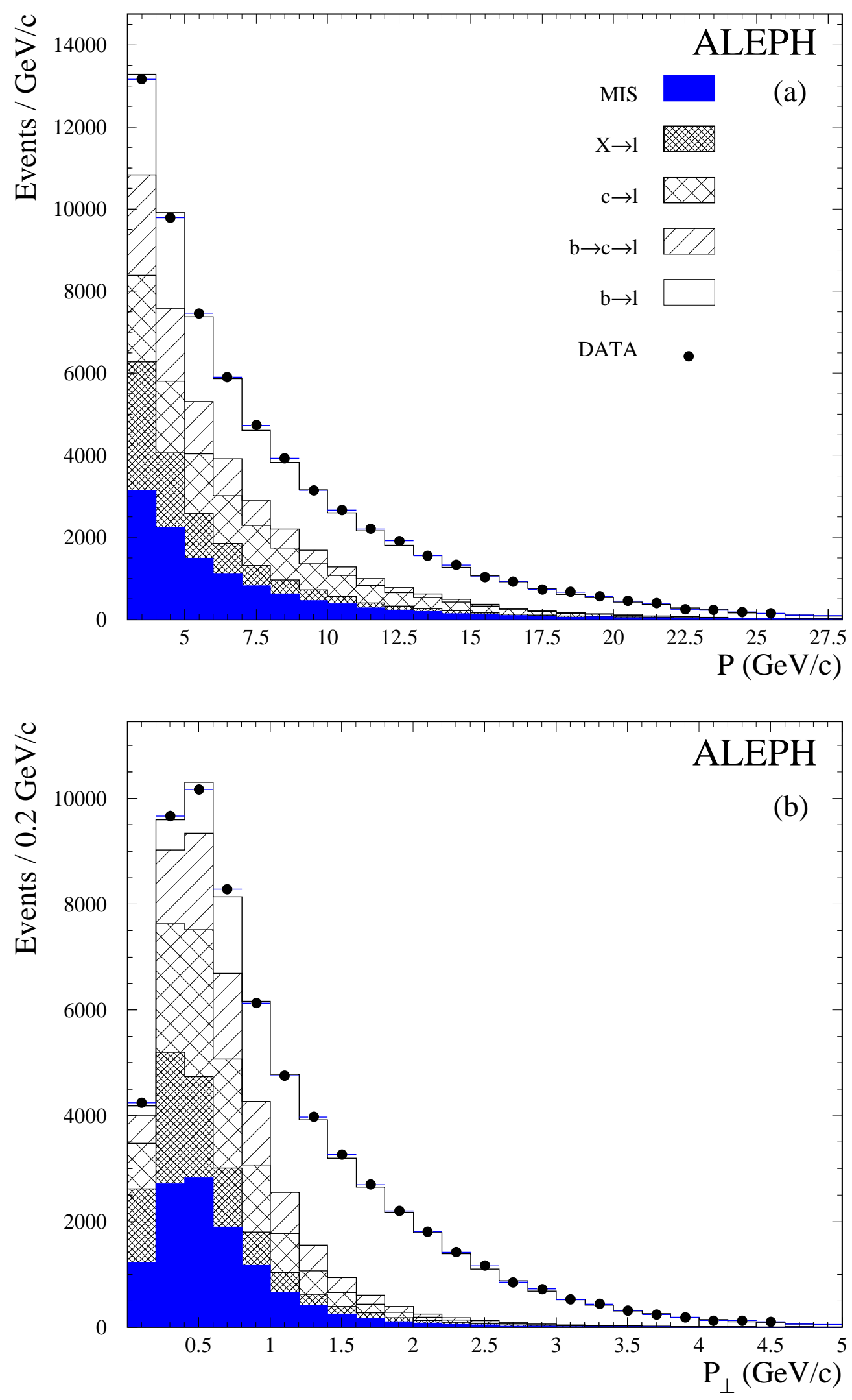

Figure 10: $p$ and $p_{\perp}$ distributions for leptons $(1=e$ and $\mu)$. 

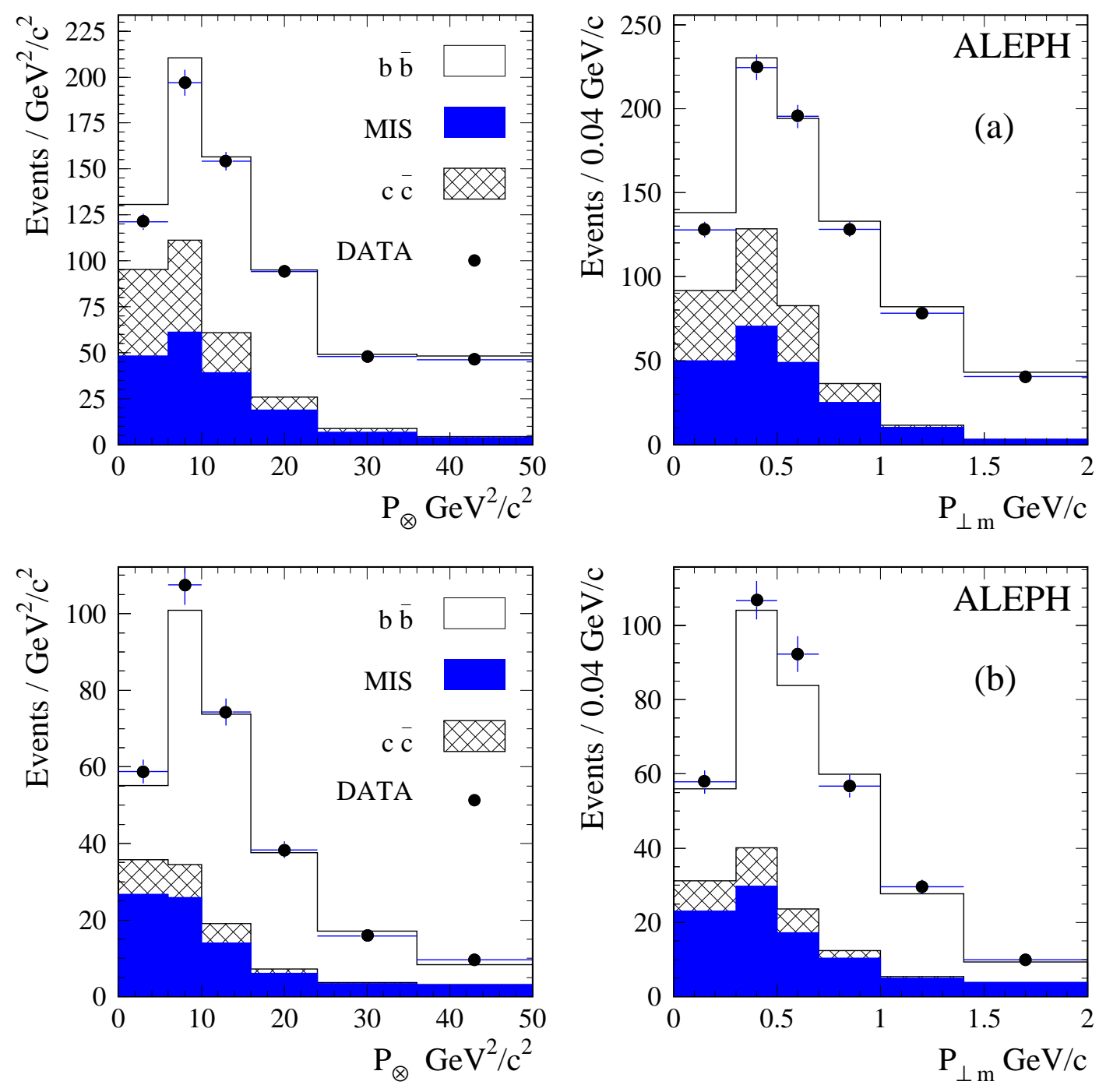

Figure 11: $P_{\otimes}$ and $P_{\perp m}$ distributions for (a) opposite direction dileptons and (b) same direction dileptons. Those $\mathrm{Z} \rightarrow b \bar{b}$ events which yield two prompt leptons from any of the processes $b \rightarrow \ell, b \rightarrow c \rightarrow \ell, b \rightarrow \tau \rightarrow \ell$, or $b \rightarrow(\bar{c} s) \rightarrow \ell$ are denoted $b \bar{b}$. The category MIS includes $b \bar{b}$ events in which any of the leptons are fake as well as any uds events. All charm events are in the category $c \bar{c}$. 


\begin{tabular}{|c|c|c|}
\hline Parameter & $\mathrm{e}$ & $\mu$ \\
\hline \hline $\mathrm{Br}\left(b \rightarrow \ell^{-}\right)$ & $0.110 \pm 0.018$ & $0.112 \pm 0.016$ \\
$\operatorname{Br}\left(b \rightarrow c \rightarrow \ell^{+}\right)$ & $0.091 \pm 0.005$ & $0.090 \pm 0.004$ \\
$\operatorname{Br}\left(c \rightarrow \ell^{+}\right)$ & $0.099 \pm 0.004$ & $0.089 \pm 0.004$ \\
$<x_{b}>$ & $0.714 \pm 0.007$ & $0.698 \pm 0.007$ \\
$\left\langle x_{c}>\right.$ & $0.527_{-0.013}^{+0.012}$ & $0.486_{-0.010}^{+0.012}$ \\
\hline
\end{tabular}

Table 20: Comparison of e and $\mu$ results, with statistical uncertainties using the ACCMM modelling for the $b \rightarrow \ell^{-}$transition.

to the value of the effective electroweak mixing angle. As described in sections 5.4 and 6 the $b$ asymmetry has been measured at seven energy points. The electroweak mixing angle has been computed by taking the point at the peak from the global analysis (which has a smaller uncertainty) and the six off peak points from the high $p_{\perp}$ measurement. Corrections for the following effects were applied to the measured $b$ and $c$ asymmetries in order to convert them to the tree level asymmetries at the $\mathrm{Z}$ mass, $A_{F B}^{0}$ :

- QED initial state radiation.

- QED final state radiation.

- The effect of photon exchange and the photon-Z interference.

- First order final state QCD corrections, as computed in [26] have been applied.

- The Standard Model energy dependence has been assumed. For the peak point, the correction is due to the difference between $\mathrm{M}_{Z}$ and the LEP energy at peak.

The relative values of the corrections are given in table 21, yielding

$$
\begin{aligned}
& A_{F B}^{0}(b)=0.090 \pm 0.013 \pm 0.003 \\
& A_{F B}^{0}(c)=0.111 \pm 0.021 \pm 0.018
\end{aligned}
$$

where the first uncertainty is statistical, and the second accounts for systematic and modeling effects.

From the tree level asymmetries the ratio between the vector and axial-vector coupling constants $g_{V}$ and $g_{A}$ is readily calculated since at the peak of the $\mathrm{Z}$ resonance 


\begin{tabular}{|l|c|c|}
\hline \multirow{2}{*}{\multicolumn{1}{|c|}{ Effects }} & \multicolumn{2}{|c|}{ Relative corrections } \\
\cline { 2 - 3 } & b asymmetry & c asymmetry \\
\hline \hline QED I.S.R. & $+4.6 \%$ & $+10 \%$ \\
QED F.S.R. & $+0.02 \%$ & $+0.08 \%$ \\
$\gamma$ exch. and $\gamma-Z$ interf. & $+0.06 \%$ & $+0.6 \%$ \\
Final state QCD corr. & $+2.3 \%$ & $+2.9 \%$ \\
Energy corr. at peak & $-0.7 \%$ & $-1.5 \%$ \\
\hline
\end{tabular}

Table 21: Relative corrections to the experimental asymmetries.

$$
A_{F B}^{0}(f)=\frac{3}{4} \mathcal{A}_{e} \mathcal{A}_{f}
$$

where

$$
\mathcal{A}_{f}=\frac{g_{L_{f}}^{2}-g_{R_{f}}^{2}}{g_{L_{f}}^{2}+g_{R_{f}}^{2}}=2 \frac{g_{V_{f}} g_{A_{f}}}{g_{V_{f}}^{2}+g_{A_{f}}^{2}} .
$$

Within the Standard Model this is related to the effective electroweak mixing angle through the relation

$$
g_{V_{f}} / g_{A_{f}}=1-\frac{2 Q_{f}}{T_{f}^{3}}\left(\sin ^{2} \theta_{W}^{e f f}+C_{f}\right)
$$

where $Q_{f}$ is the fermion charge and $T_{f}^{3}$ is the third component of the fermion weak isospin. The residual vertex correction, $C_{f}$, is equal to zero by definition for leptons. For quarks, it is small and has very little dependence on electroweak parameters [27]. The sensitivity to $\sin ^{2} \theta_{W}^{e f f}$ in equation (3) is almost entirely due to the electron coupling $\mathcal{A}_{e}$. The result for the $b$ asymmetry is

$$
\sin ^{2} \theta_{W}^{e f f}=0.2340 \pm 0.0023
$$

where the statistical, systematic and modelling uncertainties have been added in quadrature. The seven measured values are shown in figure 9, as well as the curve from the Standard Model fit of the data superimposed.

The same procedure can be applied to the $c$ asymmetry, measured in section 6 , yielding ${ }^{2}$

$$
\sin ^{2} \theta_{W}^{e f f}=0.2232 \pm 0.0062 .
$$

\footnotetext{
${ }^{2}$ In reference [28], this measurement was combined with the result from [25], giving $\sin ^{2} \theta_{W}^{e f f}=$ $0.2257 \pm 0.0053$.
} 
In combining these two measurements the $22 \%$ correlation between the $b$ and $c$ peak asymmetry has been taken into account (see table 19). This gives a total $20 \%$ correlation when the off peak points are included, giving

$$
\sin ^{2} \theta_{W}^{e f f}=0.2333 \pm 0.0022 .
$$

The energy dependence of the $b$ quark forward-backward asymmetry is almost entirely due to photon- $Z$ interference, and is therefore proportional to the linear sum over colours of the $b$ quark charge. This dependence can be used to extract a measurement of the linear sum, as distinct from the quadratic sum that is measured by the change in $\mathrm{R}$ at the $b$ quark threshold. Further, it is the linear sum that is relevant to the cancellation of the triangle anomaly [29].

The EXPOSTAR Standard Model fitting program was modified to rescale the $b$ quark charge at the Born level as a fit parameter. In this way the loop and vertex corrections were not affected and the determination only depends on the energy dependence of the interference. Fitting the measurements, allowing all other electroweak parameters $\left(\mathrm{M}_{Z}, \mathrm{M}_{\text {top }}, \alpha_{s}\right)$ to vary within their ranges results in the summed $b$ quark charge being

$$
\sum_{\text {colours }} q_{b}=-1.40 \pm .56
$$

\section{Conclusions}

In 431,000 hadronic $\mathrm{Z}$ decays recorded with the ALEPH detector at LEP, the yields of electrons and muons have been analysed to measure the observables $R_{b}=$ $\Gamma(b \bar{b}) / \Gamma(h a d), R_{c}=\Gamma(c \bar{c}) / \Gamma(h a d), A_{F B}^{0}(b)$ and $A_{F B}^{0}(c)$, which yield information on the neutral electroweak couplings of the $b$ and $c$ quarks. The branching ratios $\operatorname{Br}\left(b \rightarrow \ell^{-} \bar{\nu} \mathrm{X}\right)$ and $\operatorname{Br}\left(b \rightarrow c \rightarrow \ell^{+} \nu \mathrm{X}\right)$, the integrated $b$ mixing parameter $\chi$, and $\langle x\rangle=E_{\text {hadron }} / E_{\text {quark }}$ for both $b$ and $c$ production have also been measured. The effect of different semileptonic decay models has been allowed for in the systematic uncertainties.

In the $b$ sector these quantities are derived from analyses in both the high $p_{\perp}$ region, with a sample of events highly enriched in primary $b$ decays, and from a global fit over the whole $p_{\perp}$ region. The global analysis allows more events to be effectively used and requires less external input. As a result, the overall errors are lower than for the high $p_{\perp}$ analyses. Consequently the results are taken from the overall fit, with the following exception: the statistics available for the offpeak measurements of the $b$ asymmetry are inadequate to justify the use of an overall fit and so these measurements are taken from the high $p_{\perp}$ analyses. Where results are obtained with both analyses, no differences are observed which cannot be justified on either statistical grounds or on different sensitivities to the effect of decay models. 
The electroweak results from the multilepton fit, after QCD and QED corrections, are:

$$
\begin{aligned}
A_{F B}^{0}(b) & =0.090 \pm 0.013 \pm 0.003 \\
A_{F B}^{0}(c) & =0.111 \pm 0.021 \pm 0.018 \\
R_{b} & =0.219 \pm 0.006 \pm 0.005 \\
R_{c} & =0.165 \pm 0.005 \pm 0.020
\end{aligned}
$$

where the first uncertainty is statistical, and the second is the sum in quadrature of systematic uncertainties with the uncertainty resulting from imperfect modelling of the $b \rightarrow \ell^{-}, b \rightarrow c \rightarrow \ell^{+}$, and $c \rightarrow \ell^{+}$spectra.

From the $b$ and $c$ asymmetries at the peak and the $b$ asymmetry at the six off peak points, the electroweak mixing angle, $\sin ^{2} \theta_{W}^{e f f}$, has been measured to be

$$
\sin ^{2} \theta_{W}^{e f f}=0.2333 \pm 0.0022 .
$$

ALEPH has also measured $R_{b}$ with both a lifetime tag [30] and an event shape tag method [31]. Allowing for the correlations between the methods the combined ALEPH measurement for $R_{b}$ is

$$
R_{b}=0.2206 \pm 0.0031 \text {. }
$$

The integrated mixing rate in the $b$ system has been measured to be

$$
\chi=0.114 \pm 0.014 \pm 0.008 .
$$

The $b$ semileptonic branching ratio is known to be lower than simple spectator predictions [13] and is sensitive to strong interaction corrections. Currently few measurements exist for the semileptonic cascade rate. The present analysis yields, for the mix of $b$ hadrons produced in Z decay

$$
\begin{aligned}
B r\left(b \rightarrow \ell^{-} \bar{\nu} X\right) & =0.114 \pm 0.003 \pm 0.004 \\
\operatorname{Br}\left(b \rightarrow c \rightarrow \ell^{+} \nu X\right) & =0.082 \pm 0.003 \pm 0.012 .
\end{aligned}
$$

As the global analysis fits the momentum as well as transverse momentum distributions, a parametrisation of the momentum dependence of the $b$ hadrons is necessary. The analysis employs the model developed by Peterson et al. [18], which has one free parameter per quark species. This can be expressed in terms of the mean $x$ for that species. The results are: 


$$
\begin{aligned}
& <x_{b}>=0.714 \pm 0.004 \pm 0.011 \\
& <x_{c}>=0.487 \pm 0.008 \pm 0.008
\end{aligned}
$$

The results presented here are consistent with previous measurements from LEP experiments $[30,31,32]$.

\section{Acknowledgments}

We thank our colleagues in the accelerator divisions for the continued good performance of LEP. Thanks also to the many engineering and technical personnel at CERN and at the home institutes for their contributions to the performance of the ALEPH detector. Those of us from non-member states thank CERN for its hospitality.

\section{A Appendix: Likelihood function for the global analysis}

The total binned log likelihood of the global fit is the sum of individual log likelihoods for the three samples of events used in the analysis assuming poissonian statistical fluctuations in each bin:

$$
\mathcal{L}=\mathcal{L}^{S}+\mathcal{L}^{O S}+\mathcal{L}^{S S}
$$

where :

- $\mathcal{L}^{S}=-\ln ($ Likelihood for Lepton sample $)$

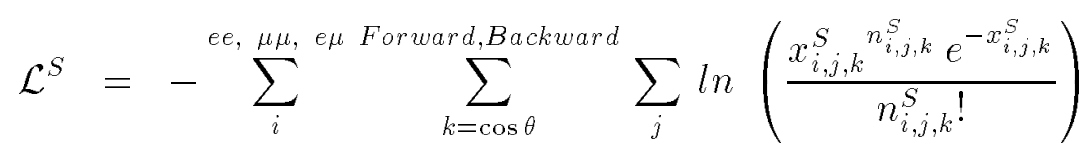

$x_{j}^{S}=N S\left(p, p_{\perp}, \theta_{t}\right):$ predicted number of leptons in bin $j$,

$n_{j}^{S}=$ the observed number of leptons in bin $j$.

- $\mathcal{L}^{O S}=-\ln$ ( Likelihood for opposite side dilepton sample)

$$
\mathcal{L}^{O S}=-\sum_{i}^{e e, \mu \mu, \text { eн same sign, opposite sign }} \sum_{k} \sum_{j} \ln \left(\frac{x_{i, j, k}^{O S} n_{i, j, k}^{O S} e^{-x_{i, j, k}^{O S}}}{n_{i, j, k}^{O S !} !}\right)
$$




$$
\begin{aligned}
x_{j}^{\text {OS }} \text { same sign }= & N D M S\left(P_{\otimes}, P_{\perp m}\right) \text { Predicted number of } \\
& \text { opposite side, } \\
& \text { same sign dileptons in bin } j \\
x_{j}^{O S} \text { opposite sign }= & N D O S\left(P_{\otimes}, P_{\perp m}\right) \text { Predicted number of } \\
& \text { opposite side, } \\
& \text { opposite sign dileptons in bin } j \\
n_{j}^{\text {OS }} \text { same sign, opposite sign }= & \text { the observed numbers }
\end{aligned}
$$

- $\mathcal{L}^{M S}=-\ln ($ Likelihood for same side dilepton sample $)$

$$
\begin{aligned}
\mathcal{L}^{M S} & =-\sum_{i}^{e e,} \sum_{j} \ln \frac{x_{i, j}^{M S} x_{i, j}^{M S} e^{-x_{i, j}^{M S}}}{x_{i, j}^{M S !}} \\
x_{j}^{M S} & =N D S S\left(P_{\otimes}, P_{\perp m}\right) \text { predicted number of same side dilepton } \\
n_{j}^{M S} & =\text { the observed number }
\end{aligned}
$$

The number of leptons in a $\left(p, p_{\perp}, \theta_{t}\right)$ box is given by:

$$
\begin{aligned}
N S\left(p, p_{\perp}, \theta_{t}\right)=(\quad 2 & R_{b}\left[\left(f_{b \rightarrow \ell}\left(p, p_{\perp}, \theta_{t}, \varepsilon_{b}\right) \operatorname{Br}(b \rightarrow \ell)\right.\right. \\
& +f_{b \rightarrow \tau \rightarrow \ell}\left(p, p_{\perp}, \theta_{t}, \varepsilon_{b}\right) \operatorname{Br}(b \rightarrow \tau \rightarrow \ell) \\
& \left.+f_{b \rightarrow(\bar{c} s) \rightarrow \ell}\left(p, p_{\perp}, \theta_{t}, \varepsilon_{b}\right) \operatorname{Br}(b \rightarrow(\bar{c} s) \rightarrow \ell)\right) A_{b}\left(\theta_{t}\right) \\
& \left.+f_{b \rightarrow c \rightarrow \ell}\left(p, p_{\perp}, \theta_{t}, \varepsilon_{b}\right) \operatorname{Br}(b \rightarrow c \rightarrow \ell) A_{b}\left(-\theta_{t}\right)\right] \\
+ & \left.2 R_{c} f_{c \rightarrow \ell}\left(p, p_{\perp}, \theta_{t}, \varepsilon_{c}\right) \operatorname{Br}(c \rightarrow \ell) A_{c}\left(\theta_{t}\right)\right) \times N_{Z} \times \epsilon\left(p, p_{\perp}, \theta\right) \\
+ & N_{q \bar{q}}\left(p, p_{\perp}, \theta_{t}\right) f_{q \bar{q} \rightarrow \text { non prompt lepton }}\left(p, p_{\perp}, \theta_{t}\right)
\end{aligned}
$$

where $N_{Z}$ is the number of $\mathrm{Z}$ hadronic events used in the analysis, $\theta_{t}$ is the signed angle between the lepton and the thrust axis of the event, $\epsilon\left(p, p_{\perp}, \theta\right)$ is the detection efficiency of a $\left(p, p_{\perp}, \theta\right)$ lepton, $\theta$ being the polar angle of the lepton, $f_{\text {process }}\left(p, p_{\perp}, \theta_{t}, \varepsilon\right)$ is the probability that a lepton from some process with fragmentation parameter $\varepsilon_{b / c}$ fills the $\left(p, p_{\perp}, \theta_{t}\right)$ box, $N_{q \bar{q}}\left(p, p_{\perp}, \theta_{t}\right)$ being the number of background events in the bin, and $R_{q}$ is the ratio $\Gamma(Z \rightarrow q \bar{q}) / \Gamma(Z \rightarrow$ hadrons $)$. 
The number of same sign dileptons in a $\left(P_{\otimes}, P_{\perp m}\right)$ box is given by:

$$
\begin{aligned}
N D M S\left(P_{\otimes}, P_{\perp m}\right)= & 2 \chi(1-\chi) N B S O\left(P_{\otimes}, P_{\perp m}\right) \\
& +(1-2 \chi(1-\chi)) N B M S\left(P_{\otimes}, P_{\perp m}\right) \\
& +N F A K E_{\text {same sign }}\left(P_{\otimes}, P_{\perp m}\right)
\end{aligned}
$$

where :

$$
\begin{aligned}
& N B S O\left(P_{\otimes}, P_{\perp m}\right)=N_{Z} \times \epsilon\left(p, p_{\perp}, \theta\right) R_{b}\left[B r(b \rightarrow \ell)^{2} f_{b \rightarrow \ell} b \rightarrow \ell\left(P_{\otimes}, P_{\perp m}, \varepsilon_{b}\right)\right. \\
& +\operatorname{Br}(b \rightarrow \ell) \operatorname{Br}(b \rightarrow \tau \rightarrow \ell) f_{b \rightarrow \ell b \rightarrow \tau \rightarrow \ell}\left(P_{\otimes}, P_{\perp m}, \varepsilon_{b}\right) \\
& +\operatorname{Br}(b \rightarrow \ell) \operatorname{Br}(b \rightarrow(\bar{c} s) \rightarrow \ell) \\
& f_{b \rightarrow \ell} b \rightarrow(\bar{c} s) \rightarrow \ell\left(P_{\otimes}, P_{\perp m}, \varepsilon_{b}\right) \\
& +\operatorname{Br}(b \rightarrow \tau \rightarrow \ell)^{2} f_{b \rightarrow \tau \rightarrow \ell} b \rightarrow \tau \rightarrow \ell\left(P_{\otimes}, P_{\perp m}, \varepsilon_{b}\right) \\
& +\operatorname{Br}(b \rightarrow \tau \rightarrow \ell) \operatorname{Br}(b \rightarrow(\bar{c} s) \rightarrow \ell) \\
& f_{b \rightarrow \tau \rightarrow \ell} b \rightarrow(\bar{c} s) \rightarrow \ell\left(P_{\otimes}, P_{\perp m}, \varepsilon_{b}\right) \\
& +\operatorname{Br}(b \rightarrow c \rightarrow \ell)^{2} f_{b \rightarrow c \rightarrow \ell b \rightarrow c \rightarrow \ell}\left(P_{\otimes}, P_{\perp m}, \varepsilon_{b}\right) \\
& +\operatorname{Br}(b \rightarrow(\bar{c} s) \rightarrow \ell)^{2} \\
& \left.f_{b \rightarrow(\bar{c} s) \rightarrow \ell} b \rightarrow(\bar{c} s) \rightarrow \ell\left(P_{\otimes}, P_{\perp m}, \varepsilon_{b}\right)\right] \\
& \operatorname{NBMS}\left(P_{\otimes}, P_{\perp m}\right)=N_{Z} \times \epsilon\left(p, p_{\perp}, \theta\right) R_{b} \\
& {\left[\operatorname{Br}(b \rightarrow \ell) \operatorname{Br}(b \rightarrow c \rightarrow \ell) f_{b \rightarrow \ell} b \rightarrow c \rightarrow \ell\left(P_{\otimes}, P_{\perp m}, \varepsilon_{b}\right)\right.} \\
& +\operatorname{Br}(b \rightarrow \tau \rightarrow \ell) \operatorname{Br}(b \rightarrow c \rightarrow \ell) f_{b \rightarrow \tau \rightarrow \ell} b \rightarrow c \rightarrow \ell\left(P_{\otimes}, P_{\perp m}, \varepsilon_{b}\right) \\
& +\operatorname{Br}(b \rightarrow(\bar{c} s) \rightarrow \ell) \operatorname{Br}(b \rightarrow c \rightarrow \ell) \\
& \left.f_{b \rightarrow(\bar{c} s) \rightarrow \ell b \rightarrow c \rightarrow \ell}\left(P_{\otimes}, P_{\perp m}, \varepsilon_{b}\right)\right] \\
& N F A K E\left(P_{\otimes}, P_{\perp m}\right)=N_{Z} \times \epsilon\left(p, p_{\perp}, \theta\right) R_{b}\left[B r(b \rightarrow \ell) f_{b \rightarrow \ell \text { other }}\left(P_{\otimes}, P_{\perp m}, \varepsilon_{b}\right)\right. \\
& +\operatorname{Br}(b \rightarrow \tau \rightarrow \ell) f_{b \rightarrow \tau \rightarrow \ell \text { other }}\left(P_{\otimes}, P_{\perp m}, \varepsilon_{b}\right) \\
& +\operatorname{Br}(b \rightarrow c \rightarrow \ell) f_{b \rightarrow c \rightarrow \ell \text { other }}\left(P_{\otimes}, P_{\perp m}, \varepsilon_{b}\right) \\
& \left.+\operatorname{Br}(b \rightarrow(\bar{c} s) \rightarrow \ell) f_{b \rightarrow(\bar{c} s) \rightarrow \ell \text { other }}\left(P_{\otimes}, P_{\perp m}, \varepsilon_{b}\right)\right] \\
& +N_{Z} \times \epsilon\left(p, p_{\perp}, \theta\right) R_{c}\left[B r(c \rightarrow \ell) f_{c \rightarrow \ell \text { other }}\left(P_{\otimes}, P_{\perp m}, \varepsilon_{c}\right)\right. \\
& \left.+\operatorname{Br}(c \rightarrow \ell)^{2} f_{c \rightarrow \ell c \rightarrow \ell}\left(P_{\otimes}, P_{\perp m}, \varepsilon_{c}\right)\right] \\
& +N_{q \bar{q}}\left(P_{\otimes}, P_{\perp m}\right) f_{q \bar{q} \rightarrow \text { non prompt lepton }}\left(P_{\otimes}, P_{\perp m}\right)
\end{aligned}
$$


The number of opposite sign dileptons in a $\left(P_{\otimes}, P_{\perp m}\right)$ box is given by:

$$
\begin{aligned}
N D S O\left(P_{\otimes}, P_{\perp m}\right)= & 2 \chi(1-\chi) N B M S\left(P_{\otimes}, P_{\perp m}\right) \\
& +(1-2 \chi(1-\chi)) N B S O\left(P_{\otimes}, P_{\perp m}\right) \\
& +N F A K E_{\text {opposite sign }}\left(P_{\otimes}, P_{\perp m}\right)
\end{aligned}
$$

The number of dileptons in a $\left(P_{\otimes}, P_{\perp m}\right)$ box is given by:

$$
\begin{aligned}
N D S S\left(P_{\otimes}, P_{\perp m}\right)= & N_{Z} \times \epsilon\left(p, p_{\perp}, \theta\right) R_{b} \\
& {\left[B r(b \rightarrow \ell) B r(b \rightarrow c \rightarrow \ell) f_{b \rightarrow \ell \rightarrow \rightarrow c \rightarrow \ell}\left(P_{\otimes}, P_{\perp m}, \varepsilon_{b}\right)\right.} \\
+ & B r(b \rightarrow \tau \rightarrow \ell) B r(b \rightarrow c \rightarrow \ell) f_{b \rightarrow \tau \rightarrow \ell} \rightarrow \rightarrow \rightarrow \ell \\
+ & B r(b \rightarrow(\bar{c} s) \rightarrow \ell) B r(b \rightarrow c \rightarrow \ell) \\
& f_{b \rightarrow(\bar{c} s) \rightarrow \ell \rightarrow c \rightarrow \ell}\left(P_{\otimes}, P_{\perp m}, \varepsilon_{b}\right) \\
+ & B r(b \rightarrow \ell) f_{b \rightarrow \ell \text { other }}\left(P_{\otimes}, P_{\perp m}, \varepsilon_{b}\right) \\
+ & B r(b \rightarrow \tau \rightarrow \ell) f_{b \rightarrow \tau \rightarrow \ell \text { other }}\left(P_{\otimes}, P_{\perp m}, \varepsilon_{b}\right) \\
+ & B r(b \rightarrow c \rightarrow \ell) f_{b \rightarrow c \rightarrow \ell \text { other }}\left(P_{\otimes}, P_{\perp m}, \varepsilon_{b}\right) \\
+ & \left.B r(b \rightarrow(\bar{c} s) \rightarrow \ell) f_{b \rightarrow(\bar{c} s) \rightarrow \ell \text { other }}\left(P_{\otimes}, P_{\perp m}, \varepsilon_{b}\right)\right] \\
+ & N_{Z} \times R_{c} \times \epsilon\left(p, p_{\perp}, \theta\right) B r(c \rightarrow \ell) f_{c \rightarrow \ell \text { other }}\left(P_{\otimes}, P_{\perp m}, \varepsilon_{c}\right) \\
+ & N_{q \bar{q}}\left(P_{\otimes}, P_{\perp m}\right) f_{q \bar{q} \rightarrow \text { on prompt lepton }}\left(P_{\otimes}, P_{\perp m}\right)
\end{aligned}
$$

\section{References}

[1] ALEPH Coll., D. Decamp et al. , Heavy Quark Tagging with Leptons in the ALEPH detector, to be published, Nucl. Instr. Methods.

[2] ALEPH Coll., D. Decamp et al., Nucl. Instr. Methods. A294 (1990) 121 ibid., A303 (1991) 393

[3] W.B. Atwood, et al. , Nucl. Instr. Methods. A306 (1991) 446

[4] T. Sjostrand and M. Bengtsson, The LUND Monte Carlo Programs, CERN long write-up, 1 November 1989

[5] ALEPH Coll., D. Buskulic et al. , Z. Phys. C55 (1992) 209

[6] J.E. Campagne and R. Zitoun, Z. Phys. C43 (1989) 469

[7] Particle Data Group, Phys. Rev. D 45 (1992) Nr. 11

CLEO Coll., D.S. Akerib, et al. , CLNS 92/1163 
[8] Particle Data Group, Phys. Rev. D 45 (1992) Nr. 11

CLEO Coll., D. Bortollo, et al. , Phys. Rev. D 45 (1992) 21 and references therein.

[9] M. Bauer, B. Stech, M. Wirbel, Z. Phys. C34 (1987) 103 M. Bauer, B. Stech, M. Wirbel, Z. Phys. C29 (1985) 637

[10] H. Schröder, Rept. Prog. Phys. 52 (1989) 765

ARGUS Coll., H. Albrecht, et al. , Z. Phys. C42 (1989) 519

[11] J.G. Körner et al. , Z. Phys. C38 (1988) 511 ibid., C41 (1989) 690.

[12] ARGUS Coll., H. Albrecht, et al. , Phys. Lett. B249 (1990) 359 CLEO Coll., S. Henderson, et al. , Phys. Rev. D45 (1992) 2212

[13] G. Altarelli et al. , Nucl. Phys. B208 (1982) 365 G. Altarelli and S. Petrarca, Phys. Lett. B261 (1991) 303

[14] M.A. Worris PhD Thesis, Cornell University, 1991 ARGUS Coll., H. Albrecht, et al. , Z. Phys. C57 (1993) 533 CLEO Coll., R. Poling, Semileptonic B decays, Contributed paper to the $X^{\text {th }}$ International Symposium on Heavy Flavour Physics, 6-10 July 1993, Montreal.

[15] B. Grinstein, et al. , Phys. Rev. D 39 (1989) 799

B. Grinstein, et al. , Phys. Rev. Lett. 56 (1986) 298

B. Grinstein, et al. , CALT-68-1311

[16] DELCO Coll., W. Bacino et al. , Phys. Rev. Lett. 43 (1979) 1073

[17] E. Barberio, et al. , CERN-TH-5857/90

D. Atwood and W.J. Marciano Phys. Rev. D 41 (1990) 1736

[18] C. Peterson, et al. , Phys. Rev. D 27 (1983) 105

[19] V.G. Kartvelishvili, et al. , Phys. Lett. B78 (1978) 615

[20] C. Quigg and J.L. Rosner, Phys. Rev. D 19 (1979) 1532

[21] ALEPH Coll., D. Buskulic et al. , Phys. Lett. B298 (1993) 479

[22] ARGUS Coll., H. Albrecht et al., Phys. Lett. B278 (1992) 202 and references therein.

[23] D. Levinthal et al. , Z. Phys. C53 (1992) 617 
[24] MARK II Coll., A.J. Weir et al., Phys. Lett. B240 (1990) 289.

[25] ALEPH Coll., D. Buskulic et al. , to be published, Z. Phys. PPE-93-208, (1993)

[26] A. Djouadi, J.H. Kühn, and P.M. Zerwas Z. Phys. C46 (1990) 411

G. Altarelli and B. Lampe, Nucl. Phys. B391 (1993) 3

[27] W. Hollik, private communication.

[28] ALEPH Coll., D. Buskulic et al. , Z. Phys. C60 (1993) 71

[29] T.P. Cheng, L.F. Li, Gauge Theory of Elementary Particle Theory, Oxford Science Publications and references therein, pg 347

[30] ALEPH Coll., D. Buskulic et al. , Phys. Lett. B313 (1993) 535.

[31] ALEPH Coll., D. Buskulic et al. , Phys. Lett. B313 (1993) 549.

[32] ALEPH Coll., D. Decamp et al. , Phys. Lett. B244 (1990) 551.

ALEPH Coll., D. Decamp et al. , Phys. Lett. B258 (1991) 237.

ALEPH Coll., D. Decamp et al. , Phys. Lett. B263 (1991) 325.

ALEPH Coll., D. Decamp et al. , Phys. Lett. B284 (1992) 177.

DELPHI Coll., P.Abreu et al. , Phys. Lett. B276 (1992) 536.

DELPHI Coll., P.Abreu et al. , Z. Phys. C56 (1992) 47.

DELPHI Coll., P.Abreu et al. , Phys. Lett. B295 (1992) 383.

DELPHI Coll., P.Abreu et al. , Phys. Lett. B301 (1993) 145.

L3 Coll., B. Adeva et al., Phys. Lett. B252 (1990) 713.

L3 Coll., B. Adeva et al. , Phys. Lett. B261 (1991) 177.

L3 Coll., B. Adeva et al. , Phys. Lett. B288 (1992) 395.

L3 Coll., B. Adeva et al., Phys. Lett. B292 (1992) 454.

L3 Coll., B. Adeva et al. , Phys. Lett. B307 (1993) 237.

OPAL Coll., P. Acton et al. , Z. Phys. C58 (1993) 523.

OPAL Coll., P. Acton et al. , Z. Phys. C60 (1993) 19.

OPAL Coll., R. Akers et al. , Z. Phys. C60 (1993) 199.

OPAL Coll., P. Acton et al. , Z. Phys. C60 (1993) 579.

OPAL Coll., R. Akers et al. , Z. Phys. C60 (1993) 601. 